(C) 2001 International Press

Adv. Theor. Math. Phys. 5 (2001) 969-999

\title{
Mathematical Analysis of
}

\section{the Photoelectric Effect}

\author{
Volker Bach, Frédéric Klopp, and Heribert Zenk
}

FB Mathematik; Johannes Gutenberg-Universität; D-55099 Mainz; Germany; vbach@mathematik.uni-mainz.de

LAGA, Institut Galilée; Université Paris-Nord; F-93430 Villetaneuse; France klopp@math.univ-paris13.fr

FB Mathematik; Johannes Gutenberg-Universität; D-55099 Mainz; Germany; zenk@mathematik.uni-mainz.de

\begin{abstract}
We study the photoelectric effect on the example of a simplified model of an atom with a single bound state, coupled to the quantized electromagnetic field.

For this model, we show that Einstein's prediction for the photoelectric effect is qualitatively and quantitatively correct to leading order in the coupling parameter. More specifically, considering the ionization of the atom by an incident photon cloud consisting of $N$ photons, we prove that the total ionized charge is additive in the $N$ involved photons. Furthermore, if the photon cloud is approaching the atom from a large distance, the kinetic energy of the ejected electron is shown to be given by the difference of the photon energy of each single photon in the photon cloud and the ionization energy.
\end{abstract}

MSC: 81Q10, 81V10, 47N50.

Keywords: Photoelectric Effect, Scattering Theory, QED. 


\section{Introduction}

The photoelectric effect was discovered in increasingly precise experiments by Hertz [17, 1887], Hallwachs [16, 1888], Lenard [18, 1902] ${ }^{1}$, and Millikan $[21,20,1916]$.

It was observed that, when light is incident on a metal surface, electrons are ejected from the surface. The striking fact about this phenomenon is the seemingly odd dependence of the maximal kinetic energy $T_{\max }$ of the ejected electrons on the frequency of the light and its independence of the light intensity. The latter contradicts the principles of classical physics, and in 1905, Einstein suggested an explanation of this phenomenon $[9,1905]$ which explicitly involves the quantum nature of electromagnetic radiation. He found that

$$
T_{\max }=h \nu-\Delta E,
$$

provided that the frequency $\nu$ of the light times Planck's constant $h$, i.e., the photon energy $h \nu$ is larger than the (material dependent) work function $\Delta E$. Conversely, if

$$
h \nu<\Delta E,
$$

then no electrons leave the metal surface. Our ultimate goal is the derivation of Einstein's predictions, Eqs. (I.1)-(I.2), from first principles of quantum mechanics and quantum field theory.

In the present paper, we analyze a simplified model which is far from the appropriate model for a metal interacting with electromagnetic radiation and can, at best, be regarded as a caricature of a hydrogen atom interacting with the radiation field. Yet, it contains many of the mathematical difficulties we expect to encounter in the analysis of a more realistic model, and we prove Eqs. (I.1)-(I.2) for this simplified model. Our emphasis lies in the following aspects:

- Given the model as described in Sect. I.1, below, our derivation is mathematically rigorous, and no unjustified approximations are used. To our knowledge, the present paper is the first to treat the photoelectric effect with mathematical rigor.

We draw from many facts about nonrelativistic quantum electrodynamics which have been previously established in $[2,3,4,5]$.

As we propose to study the charge transported to infinity (see Sect. I.2) which involves the asymptotics of the unitary time evolution operator $e^{-i t H_{g}}$, as $t \rightarrow \infty$, our results can be viewed as part of scattering theory for models of nonrelativistic quantum electrodynamics. Results in this context, but on other aspects can be found in $[1,6,10,12,13,23]$.

- While our model for the particle system, i.e., the metal or atom, is a crude model involving only a single, spinless electron, the particle system is coupled to a quantized scalar field. (The difference between the quantized [vector] electromagnetic and a quantized scalar field is irrelevant, for the scope of this work. For certain other facts in nonrelativistic quantum electrodynamics, however, this difference is crucial, see, e.g., [4].)

\footnotetext{
${ }^{1}$ In some physics textbooks, e.g., [15], Lenard is not mentioned, and it seems that in 1905, Einstein derived his famous theory from nothing but a Gedankenexperiment.
} 
- Our model describes a single atom rather than a metal or even a gas interacting with the electromagnetic field. Thus our derivation of Eqs. (I.1)-(I.2) shows that the photoelectric effect is not a collective, statistical phenomenon, visible only if many particles or many photons are involved. Most texts on laser theory or quantum optics, e.g., $[8,14]$, immediately proceed to a statistical description of both the metal or gas of atoms, say, and the photon field, and the question, whether this is really necessary or merely a matter of mathematical convenience, is left open.

- Within our framework, we prove Eqs. (I.1)-(I.2) to be correct in leading nonvanishing order in the coupling parameter $g$ which, in appropriate units, equals $\sqrt{2 \pi} \alpha^{3 / 2}$, where $\alpha \approx 1 / 137$ is the fine structure constant. More precisely, given the interacting atom at rest plus an incident photon cloud consisting of $N$ photons, we show that to leading order the contribution to the charge ejected from the atom is additive in each photon and independent of all other photons of the incident photon cloud. Moreover, we prove that this contribution is in accordance with (I.1)-(I.2) in case that the photons are in an incoming scattering state. In fact, the leading order contribution to the ejected charge resembles the first term in the Born series for the T-Matrix (see, e.g., [22]).

- While it is customary to restrict the analysis of the photoelectric effect to a single photon scattering off the atom or metal, we point out that it is important to consider more than one photon, $N \geq 2$, because for a photon state consisting of a single photon only, total energy and energy of each single photon involved agree. Hence, if studying a single photon state, it is impossible to say whether the ejection of electrons is proportional to the total energy of the photon cloud or depends on the maximal energy of all photons in the photon cloud.

Our paper is organized as follows. In Sect. I.1 below we introduce the mathematical model for the photoelectric effect. This includes a precise description of the atom, the photons, and their interaction in terms of a semibounded, selfadjoint generator of the dynamics, the Hamiltonian.

In Sect. I.2 we then describe our main results. In Subsects. I.2.1-I.2.2, we introduce the main quantities dealt with in this paper, the charge transported to infinity and photon clouds, and in Subsect. I.2.3, we derive the asymptotics of the former to leading order in the coupling constant. A limit of monochromatic light is discussed in Subsect. I.2.4, and in Subsect. I.2.5, we compare our methods and results to those derived or used in other papers on scattering theory.

The proof of the theorems in Sect. I.2 are given in detail in Sects. II and III.

Finally, our paper contains two appendices. In Appendix A we construct a Bogoliubov transformation that eliminates a single, arbitrary matrix element in the interaction, and in Appendix B we show that bound or negative energy states do not contribute to the transported charge.

Acknowledgement: We thank S. De Bièvre, J. Fröhlich, Ch. Gerard, M. Griesemer, V. Kostrykin, T. Paul, B. Schlein, R. Schrader, I. M. Sigal, H. Spohn, and S. Teufel for helpful discussions and remarks. 
We gratefully acknowledge the support of our project by the TMR network ERBFMRXCT960001 of the European Union and the Gerhard Hess-grant Ba 1477/3-1 of the german science foundation DFG.

\section{I.1 Mathematical Model for the Photoelectric Effect}

Our goal is to analyze the photoelectric effect on the example of an atom coupled to the quantized radiation field. The mathematical model for our analysis of the photoelectric effect is a variant of the standard model of nonrelativistic quantum electrodynamics as given in [2] which we briefly recall and adapt to our problem at hand.

\section{I.1.1 Atom with a Single Bound State}

For the atom to be described we make the simplifying assumptions that it consists of a single, spinless electron bound in a potential well which admits exactly one bound state of energy $e_{0}<0$. More specifically, we assume the Hamiltonian generating the dynamics of the electron to be given in diagonal form as

$$
H_{e l}=-\Delta \oplus e_{0}=\left(\begin{array}{cc}
-\Delta & 0 \\
0 & e_{0}
\end{array}\right)
$$

acting on

$$
\mathcal{H}_{e l}:=L^{2}\left(\mathbb{R}^{3}\right) \oplus \mathbb{C}=\mathcal{H}_{a c}\left(H_{e l}\right) \oplus \mathcal{H}_{d}\left(H_{e l}\right),
$$

and $\mathcal{H}_{s c}\left(H_{e l}\right)=\{0\}$. We further denote by

$$
P_{c}:=\left(\begin{array}{ll}
1 & 0 \\
0 & 0
\end{array}\right) \quad \text { and } \quad P_{d}:=P_{c}^{\perp}=\left(\begin{array}{ll}
0 & 0 \\
0 & 1
\end{array}\right)
$$

the projections onto the continuous subspace and the discrete subspace (of dimension one), respectively. The Hamiltonian $H_{e l}$ can be derived from a Schrödinger operator $-\Delta-V(x)$ with a short-range potential $V$. An appropriate choice of $V$ guarantees that $-\Delta-V(x)$ has a single bound state only, and then $-\Delta-V(x)$ and $H_{e l}$ are unitarily equivalent, as can be seen by conjugating $-\Delta-V(x)$ with the wave operators.

\section{I.1.2 Photons}

We couple the atom described above to the quantized photon field which, for notational convenience, is assumed to be scalar. For our study of the photoelectric effect, the difference is not relevant. The Hilbert space $\mathcal{F}$, carrying the photon degrees of freedom is the bosonic Fock space $\mathcal{F}=\mathcal{F}_{b}\left[L^{2}\left(\mathbb{R}^{3}\right)\right]$ over the one-photon Hilbert space $L^{2}\left(\mathbb{R}^{3}\right)$, i.e.,

$$
\mathcal{F}=\bigoplus_{n=0}^{\infty} \mathcal{F}^{(n)}
$$


where $\mathcal{F}^{(n)}$ is the state space of all $n$-photon states, the $n$-photon sector. The vacuum sector, $\mathcal{F}^{(0)}$, is one-dimensional and spanned by the normalized vacuum vector, $\Omega$, i.e., $\mathcal{F}^{(0)}:=\mathbb{C} \Omega$. For $n \geq 1$, the $n$-photon sector is the subspace of $L^{2}\left[\left(\mathbb{R}^{3}\right)^{n}\right]$ containing all totally symmetric vectors. The Hamiltonian on $\mathcal{F}$ representing the energy of the free photon field is given by

$$
H_{f}=\int d^{3} k \omega(k) a^{*}(k) a(k)
$$

where $\omega(k):=|k|$ is the photon dispersion, and $a^{*}, a$ are the usual standard creation- and annihilation operators on $\mathcal{F}$ representing the canonical commutation relations, $\left[a(k), a\left(k^{\prime}\right)\right]$ $=\left[a^{*}(k), a^{*}\left(k^{\prime}\right)\right]=0,\left[a(k), a^{*}\left(k^{\prime}\right)\right]=\delta\left(k-k^{\prime}\right), a(k) \Omega=0$, in the sense of operator-valued distributions.

\section{I.1.3 The Atom-Photon System}

The Hilbert space of states of the atom-photon system is the tensor product space

$$
\mathcal{H}:=\mathcal{H}_{e l} \otimes \mathcal{F}
$$

and its dynamics is generated by the Hamiltonian

$$
H_{g}:=H_{0}+g W,
$$

where

$$
H_{0}:=H_{e l} \otimes \mathbf{1}_{f}+\mathbf{1}_{e l} \otimes H_{f}
$$

is the non-interacting Hamiltonian, $0<g \ll 1$ is a small coupling parameter, and

$$
W:=\int d^{3} k\left\{G(k) \otimes a^{*}(k)+G^{*}(k) \otimes a(k)\right\}
$$

is the interaction operator. Here, $G \in L^{2}\left[\mathbb{R}^{3} ; \mathcal{B}\left(\mathcal{H}_{e l}\right)\right]$ is a square-integrable function with values in the bounded operators on $\mathcal{H}_{e l}$, given by

$$
G(k):=\left(\begin{array}{cc}
B(k) & p_{\uparrow}(k) \\
p_{\downarrow}(k) & 0
\end{array}\right)
$$

for $k \in \mathbb{R}^{3}$, a.e. For the formulation of assumptions about $G$ it is convenient to introduce

$$
J_{K, \gamma}(k):=\max _{|\alpha| \leq K}\left\|\partial_{k}^{\alpha} G(k)\right\|+\left\|\left(|x|^{\gamma} \oplus 1\right) G(k)\right\|+\left\|G(k)\left(|x|^{\gamma} \oplus 1\right)\right\| .
$$

Note that, for $J_{K, \gamma}(k)<\infty$, we necessarily assume a decay of the coupling matrix $G(k)$ as least as $|x|^{-\gamma}$, as $x \rightarrow \infty$. This assumption is not satisfied, e.g., if we consider electrons minimally coupled to the quantized radiation field. Namely, in the case of minimal coupling, the linear part of the interaction operator is of the form (I.11), with

$$
B_{m . c .}(k)=\frac{\kappa(k) e^{i \vec{k} \cdot \vec{x}}}{i|k|^{1 / 2}} \vec{\varepsilon}(k) \cdot \vec{\nabla}_{x},
$$


where $k=(\vec{k}, \sigma) \in \mathbb{R}^{3} \times \mathbb{Z}_{2}, \kappa$ is a smooth function of rapid decay, as $|\vec{k}| \rightarrow \infty$, serving as an ultraviolet cutoff, and $\vec{\varepsilon}(\vec{k}, \pm 1) \perp \vec{k}$ are two transversal, normalized polarization vectors. Besides the lack of decay of $B_{m . c .}(k)$, as $|x| \rightarrow \infty, B_{m . c .}(k)$ is not bounded. This is, however, only a minor complication. The extension of our results to this case of main physical interest will be the subject of a forthcoming paper.

Further note that

$$
\begin{gathered}
J_{K, \gamma}(k) \leq J_{K, \gamma}^{\prime}(k):=\max _{|\alpha| \leq K}\left\|\partial_{k}^{\alpha} B(k)\right\|+\left\||x|^{\gamma} B(k)\right\|+\left\|B(k)|x|^{\gamma}\right\| \\
\quad+\max _{|\alpha| \leq K}\left\|\partial_{k}^{\alpha} p_{\uparrow}(k)\right\|+\max _{|\alpha| \leq K}\left\|\partial_{k}^{\alpha} p_{\downarrow}(k)\right\|+\left\||x|^{\gamma} p_{\uparrow}(k)\right\|+\left\|p_{\downarrow}(k)|x|^{\gamma}\right\|,
\end{gathered}
$$

and, conversely, $J_{K, \gamma}^{\prime}(k) \leq 3 J_{K, \gamma}(k)$. We shall make use of the following Hypothesis throughout the paper.

Hypothesis 1. There is an integer $K>1$ and a real number $\gamma>3 / 2$ such that $G \in$ $C^{K}\left[\mathbb{R}^{3} ; \mathcal{B}\left(\mathcal{H}_{e l}\right)\right]$ is $K$ times differentiable and satisfies the following estimate,

$$
\int\left(1+\omega(k)^{-1}\right)\left|J_{K, \gamma}(k)\right|^{2} d^{3} k \leq 1
$$

We remark that, although the requirement (I.16) with fractional derivatives of $G$, for real $K>1$, is presumably sufficient, we do not try to optimize our result in this respect and work with classical derivatives in this paper.

Since, for a.e. $k \in \mathbb{R}^{3}$,

$$
p_{\uparrow}(k): \mathbb{C} \rightarrow L^{2}\left(\mathbb{R}^{3}\right) \quad \text { and } \quad p_{\downarrow}(k): L^{2}\left(\mathbb{R}^{3}\right) \rightarrow \mathbb{C},
$$

there exist $\rho(\cdot, k), \eta(\cdot k) \in L^{2}\left(\mathbb{R}^{3}\right)$, such that

$$
\left[p_{\uparrow}(k) z\right](x)=z \eta(x, k) \text { and } p_{\downarrow}(k) \psi=\langle\rho(\cdot, k) \mid \psi\rangle=\int \overline{\rho(x, k)} \psi(x) d^{3} x,
$$

for all $z \in \mathbb{C}, \psi \in L^{2}\left(\mathbb{R}^{3}\right)$, and $(x, k) \in \mathbb{R}^{3} \times \mathbb{R}^{3}$, a.e. Furthermore, in many applications $B(k) \in \mathcal{B}\left[L^{2}\left(\mathbb{R}^{3}\right)\right]$ acts in the Schrödinger representation as a multiplication operator. That is, there is a function $M \in L^{2}\left(\mathbb{R}^{3} \times \mathbb{R}^{3}\right)$ such that

$$
[B(k) \psi](x)=M(x, k) \psi(x),
$$

for $(x, k) \in \mathbb{R}^{3} \times \mathbb{R}^{3}$, a.e. For instance, in case of the dipole approximation,

$$
M_{d i p}(x, k)=\kappa(\vec{x} / R) \kappa(k)|k|^{1 / 2} \vec{\varepsilon}(k) \cdot \vec{x},
$$

using the same notation as for $B_{m . c .}$, above, and additionally a spatial cutoff $\kappa(\vec{x} / R)$ at length scale $R \gg 1$ which should be chosen large, compared to atomic length scales. We remark that, for a given function $M$, a physically natural choice for $\eta$ and $\rho$ is

$$
\eta(x, k):=\rho(x, k):=M(x, k) \varphi_{e l}(x)
$$


where $\varphi_{e l} \in L^{2}\left(\mathbb{R}^{3}\right),\left\|\varphi_{e l}\right\|=1$, is the normalized wave function of the atomic bound state. Note that the special form (I.12) of $G(k)$ implies that

$$
\left(P_{d} \otimes \mathbf{1}\right) W\left(P_{d} \otimes \mathbf{1}\right)=0,
$$

since the lower right matrix element of $G(k)$ vanishes.

We remark that if the electron Hamiltonian is a Schrödinger operator, as described below Eq. (I.5), a single diagonal matrix element can always be "gauged away" by means of a Bogoliubov transformation. The construction of this Bogoliubov transformation is given in Appendix A. That is, for our model with a single atomic bound state, we do not loose any generality by assuming (I.22). As (I.22) is an important assumption for the present paper, we point out that, in case the atom has more than one bound state, $P_{d}$ would be the orthogonal projection onto these, and (I.22) would imply, that the field does not couple different bound states.

\section{I.1.4 Selfadjointness, Semiboundedness, and Binding}

Next, we discuss selfadjointness, semiboundedness and existence of a ground state (binding) of the Hamiltonian $H_{g}$ of the system. We basically invoke the theorems and methods for their proof established in [2, Sects. II and III].

Assuming Hypothesis 1, selfadjointness and semiboundedness, for any value of the coupling parameter $g>0$, are a simple consequence of standard Kato perturbation theory; we appeal to [2, Cor. I.7 and Lemma I.5] which yield that $H_{g}$ is selfadjoint on its natural domain, $\operatorname{dom}\left[H_{g}\right]=\operatorname{dom}\left[H_{0}\right]$, and that the ground state energy is given by

$$
E_{0}:=\inf \sigma\left[H_{g}\right] \geq e_{0}+\mathcal{O}\left(g^{2}\right)
$$

In the present paper we additionally assume that $E_{0}$ is an eigenvalue of $H_{g}$ and that the corresponding eigenvectors have a large component in the vacuum sector $\mathcal{F}^{(0)}$. More precisely, we require

Hypothesis 2. The Hamiltonian $H_{g}$ possesses a (normalizable) ground state $\Phi_{\mathrm{gs}} \in \mathcal{H}$, $\left\|\Phi_{\mathrm{gs}}\right\|=1$, i.e., $E_{0}$ is an eigenvalue with corresponding eigenvector $\Phi_{\mathrm{gs}}$,

$$
H_{g} \Phi_{\mathrm{gs}}=E_{0} \Phi_{\mathrm{gs}} .
$$

Moreover, denoting $P_{\Omega}:=|\Omega\rangle\langle\Omega|\equiv 1 \otimes| \Omega\rangle\langle\Omega|$, the ground state $\Phi_{\mathrm{gs}}$ obeys

$$
\left\|P_{\Omega}^{\perp} \Phi_{\mathrm{gs}}\right\| \leq C g
$$

for some constant $C<\infty$.

The existence (I.24) of a ground state $\Phi_{\mathrm{gs}}$ and the overlap bound (I.25) is proved in [2, Thm. I.1] under the assumption of a somewhat stronger bound than Eq. (I.16) in Hypothesis 1 , namely,

$$
\int\left(1+\omega(k)^{-2}\right)\left|J_{0, \gamma}(k)\right|^{2} d^{3} k \leq 1
$$


In this paper, we do not use this estimate but only its consequence in form of Hypothesis 2 .

We remark that the noninteracting Hamiltonian $H_{0}$ has the unique (non-degenerate) ground state $0 \oplus \Omega \in \mathcal{H}$ corresponding to its ground state energy $e_{0}$, and we note in parentheses that, by the results of [2], Eq. (I.25) holds for any ground state of $H_{g}$. Consequently, the interacting ground state $\Phi_{\text {gs }}$ of $H_{g}$ is unique, provided $g>0$ is sufficiently small.

In Lemma III. 2 we strengthen (I.25) and show that, for any $\alpha \geq 1$ and any $\Lambda<\infty$,

$$
\left\|\left(H_{f}^{(\Lambda)}+\mathbf{1}\right)^{\alpha} P_{c} \Phi_{\mathrm{gs}}\right\|+\left\|\left(H_{f}^{(\Lambda)}+\mathbf{1}\right)^{\alpha} P_{\Omega}^{\perp} \Phi_{\mathrm{gs}}\right\| \leq \mathcal{O}(g) .
$$

Here, $H_{f}^{(\Lambda)}$ denotes a free photon Hamiltonian acting only on photon states with energy less than $\Lambda<\infty$,

$$
H_{f}^{(\Lambda)}=\int d^{3} k \omega_{\Lambda}(k) a^{*}(k) a(k)=\int_{\{\omega(k) \leq \Lambda\}} d^{3} k \omega(k) a^{*}(k) a(k),
$$

where $\omega_{\Lambda}(k):=\omega(k) \mathbf{1}_{\{\omega(k)<\Lambda\}}$.

\section{I.2 Main Results and Discussion}

\section{I.2.1 Charge transported to Infinity}

Having introduced the quantum mechanical framework in terms of Hilbert spaces and Hamiltonians and established some basic facts such as selfadjointness, semiboundedness, and binding, we proceed to defining the charge transported to infinity or simply the transported charge. To this end, we introduce the projection $F_{R}$ onto the functions with support outside the ball of radius $R>0$. More precisely,

$$
F_{R}:=\left(\begin{array}{cc}
\mathbf{1}_{\{|x| \geq R\}} & 0 \\
0 & 0
\end{array}\right) \otimes \mathbf{1}_{f}
$$

where $\mathbf{1}_{\{|x| \geq R\}}:=\mathbf{1}_{\mathbb{R}^{3} \backslash B(0, R)}[x]$. Similarly, we introduce the projection $T_{\mathcal{T}}$ onto the particle states with momentum in a measurable set $\mathcal{T} \subset \mathbb{R}^{3}$, i.e., the functions whose Fourier transform is supported in $\mathcal{T}$. That is,

$$
T_{\mathcal{T}}:=\left(\begin{array}{cc}
\mathbf{1}_{\{p \in \mathcal{T}\}} & 0 \\
0 & 0
\end{array}\right) \otimes \mathbf{1}_{f}
$$

where $\mathbf{1}_{\{p \in \mathcal{T}\}}$ acts as a Fourier multiplier with $\mathbf{1}_{\mathcal{T}}[p]$. Given a state $\Psi \in \mathcal{H}$, the corresponding (least and most) transported charges with momentum in $\mathcal{T} \subset \mathbb{R}^{3}$ are defined to be

$$
\begin{aligned}
Q_{\mathcal{T}}^{\mathrm{inf}}(\Psi) & :=\liminf _{R \rightarrow \infty} \liminf _{t \rightarrow \infty}\left\|T_{\mathcal{T}} F_{R} e^{-i t H_{g}} \Psi\right\|^{2}, \\
Q_{\mathcal{T}}^{\text {sup }}(\Psi) & :=\limsup _{R \rightarrow \infty} \limsup _{t \rightarrow \infty}\left\|T_{\mathcal{T}} F_{R} e^{-i t H_{g}} \Psi\right\|^{2},
\end{aligned}
$$

In case that $\mathcal{T}=\mathbb{R}^{3}$, we write $Q^{\inf }(\Psi):=Q_{\mathcal{T}}^{\inf }(\Psi)$ and $Q^{\sup }(\Psi):=Q_{\mathcal{T}}^{\text {sup }}(\Psi)$. Interpreting $\Psi$ as an initial state for $t=0$, the transported charge $Q^{\inf }(\Psi)$ measures the amount 
of its mass that is definitely transported away from the atom as time evolves, eventually. If $\mathcal{T} \subseteq \mathbb{R}^{3}$ is a small ball, then $Q_{\mathcal{T}}(\Psi)$ additionally filters out the part of the state with momentum in $\mathcal{T}$.

While it would be desirable, of course, to define only one type of transported charge, namely, $Q_{\mathcal{T}}(\Psi):=\lim _{R \rightarrow \infty} \lim _{t \rightarrow \infty}\left\|T_{\mathcal{T}} F_{R} e^{-i t H_{g}} \Psi\right\|^{2}$, our methods described below do not allow us to prove the existence of such a limit - not even for the restricted class of initial states of the form (I.35). Nevertheless, $Q_{\mathcal{T}}^{\inf }(\Psi)$ and $Q_{\mathcal{T}}^{\text {sup }}(\Psi)$ agree to leading order in the coupling constant $g$, as we demonstrate below. In fact, our main objective is the determination of the transported charges $Q_{\mathcal{T}}^{\inf }(\Psi)$ and $Q_{\mathcal{T}}^{\text {sup }}(\Psi)$ to leading order in $g$. Finally, we remark that it is important to observe the order of the two limits $R \rightarrow \infty$ and $t \rightarrow \infty$, as interchanging these limits would, indeed, yield the trivial result $\lim _{t \rightarrow \infty} \lim _{R \rightarrow \infty}\left\|F_{R} e^{-i t H_{g}} \Psi\right\|^{2}=0$, for all $\Psi \in \mathcal{H}$.

As we prove in Appendix B,

$$
\begin{aligned}
& \lim _{R \rightarrow \infty} \sup _{t>0}\left\|F_{R} \mathbf{1}_{p p}\left(H_{g}\right) e^{-i t H_{g}} \Psi\right\|=0 \\
& \lim _{R \rightarrow \infty} \sup _{t>0}\left\|F_{R} \mathbf{1}_{\mathbb{R}_{0}^{-}}\left(H_{g}\right) e^{-i t H_{g}} \Psi\right\|=0
\end{aligned}
$$

for all $\Psi \in \mathcal{H}$, so the transported charge of all bound states $\Psi \in \operatorname{Ran} \mathbf{1}_{p p}\left(H_{g}\right)$ and of all states $\Psi \in \operatorname{Ran} \mathbf{1}_{\mathbb{R}_{0}^{-}}\left(H_{g}\right)$ of negative total energy vanishes.

\section{I.2.2 Ground State and Photon Cloud}

Our choice for the initial state $\Psi$ is of the form

$$
\Psi:=A(\tau, \underline{f}) \Phi_{\mathrm{gs}}
$$

where

$$
A(0, \underline{f}) \equiv A(\underline{f}):=\mathbf{1}_{e l} \otimes a^{*}\left(f_{1}\right) a^{*}\left(f_{2}\right) \cdots a^{*}\left(f_{N}\right)
$$

and

$$
A(\tau, \underline{f}):=e^{-i \tau H_{g}} e^{i \tau H_{0}} A(\underline{f}) e^{-i \tau H_{0}} e^{i \tau H_{g}} .
$$

The operator $A(\tau, f)$ is called a photon cloud and represents $N$ photons with corresponding smooth orbitals $\underline{f}=\left(f_{1}, f_{2}, \ldots, f_{N}\right), f_{j} \in C_{0}^{\infty}\left(\mathbb{R}^{3} \backslash\{0\}\right)$, of compact support away from zero momentum. These $N$ photons are prepared at time $t=-\tau$ in such a way that they hit the atom at rest at time $t=0$. (We thank S. De Bièvre, M. Griesemer, H. Spohn and especially S. Teufel for clarifying this point to us.) From Eq. (II.8) below we show that, in particular, $A(\infty, \underline{f}) \Phi_{\mathrm{gs}}:=\lim _{\tau \rightarrow \infty} A(\tau, f) \Phi_{\mathrm{gs}}$ exists, thus representing an incoming photon scattering state. In contrast, choosing $\bar{\Psi}$ according to (I.35) and with $\tau=0$ amounts to adding at time $t=0$ the photon cloud $A(\underline{f})$ to the (interacting) atom at rest.

We henceforth often leave out trivial tensor factors in our notation whenever it is clear from the context what is meant. For instance, we write $A(\underline{f})=a^{*}\left(f_{1}\right) a^{*}\left(f_{2}\right) \cdots a^{*}\left(f_{N}\right)$, $H_{0}=H_{e l}+H_{f}$, etc. 


\section{I.2.3 The Photoelectric Effect}

Having defined the transported charges with momentum in $\mathcal{T}$, we can now formulate a quantitative assertion about it. To this end, we fix $p \in \mathbb{R}^{3}$ and define two distributions $L_{0}^{p}, L_{\infty}^{p} \in \mathcal{D}^{\prime}\left(\mathbb{R}^{3} \backslash\{0\}\right)$ on smooth functions $f \in C_{0}^{\infty}\left(\mathbb{R}^{3} \backslash\{0\}\right)$ of compact support away from zero by

$$
\begin{aligned}
\left\langle L_{\infty}^{p}, f\right\rangle:= & 2 \pi \int \delta\left(p^{2}-E_{0}-\omega(k)\right) \hat{\rho}(p, k) f(k) d^{3} k \\
\left\langle L_{0}^{p}, f\right\rangle:= & \int \frac{-i \hat{\rho}(p, k) f(k) d^{3} k}{p^{2}-E_{0}-\omega(k)+i 0} \\
= & -\pi \int \delta\left(p^{2}-E_{0}-\omega(k)\right) \hat{\rho}(p, k) f(k) d^{3} k \\
& -i \mathbb{P} \int \frac{\hat{\rho}(p, k) f(k) d^{3} k}{p^{2}-E_{0}-\omega(k)}
\end{aligned}
$$

$\mathbb{P} \int$ denoting the Cauchy principal value and $\hat{\rho}(p, k)$ denoting the partial Fourier transform of $\rho(x, k)$ (as a square-integrable function) with respect to the particle position variable $x$.

Now we are in position to formulate our main result.

Theorem I.1. Assume Hypotheses 1-2, fix $N, m \in \mathbb{N}$ with $m \leq N$, and let $\varphi_{1}, \varphi_{2}, \ldots \varphi_{m} \in$ $C_{0}^{\infty}\left(\mathbb{R}^{3} \backslash\{0\}\right)$ be an orthonormal system, $\left\langle\varphi_{i} \mid \varphi_{j}\right\rangle=\delta_{i, j}$. Let $\bar{A}(\tau, \underline{\varphi})$ be the corresponding photon cloud, i.e.,

$$
A(\tau, \underline{\varphi})=e^{-i \tau H_{g}} e^{i \tau H_{0}} a^{*}\left(\varphi_{1}\right)^{n_{1}} a^{*}\left(\varphi_{2}\right)^{n_{2}} \cdots a^{*}\left(\varphi_{m}\right)^{n_{m}} e^{-i \tau H_{0}} e^{i \tau H_{g}},
$$

where $n_{j} \in \mathbb{N}$ are such that $n_{1}+n_{2}+\ldots+n_{m}=N$, and fix a measurable set $\mathcal{T} \subseteq \mathbb{R}^{3}$.

(i) If $\tau>g^{-1 / K}$ then the transported charges with momentum in $\mathcal{T}$ of the initial state $A(\tau, \underline{\varphi}) \Phi_{\mathrm{gs}}$ satisfy

$$
\begin{aligned}
& Q_{\mathcal{T}}^{\inf }\left(A(\tau, \underline{\varphi}) \Phi_{\mathrm{gs}}\right)+\mathcal{O}\left(g^{2+\mu}\right)=Q_{\mathcal{T}}^{\mathrm{sup}}\left(A(\tau, \underline{\varphi}) \Phi_{\mathrm{gs}}\right)+\mathcal{O}\left(g^{2+\mu}\right) \\
& \quad=g^{2} Q_{\mathcal{T}}^{\tau}(\underline{\varphi}):=g^{2}\left(n_{1} ! n_{2} ! \cdots n_{m} !\right) \sum_{j=1}^{m} n_{j} \int_{\mathcal{T}}\left|\left\langle L_{\infty}^{p}, \varphi_{j}\right\rangle\right|^{2} d^{3} p .
\end{aligned}
$$

(ii) If $\tau<g^{\mu}$ then the transported charge with momentum in $\mathcal{T}$ of the initial state $A(\tau, \underline{\varphi}) \Phi_{\mathrm{gs}}$ is given by

$$
\begin{aligned}
& Q_{\mathcal{T}}^{\inf }\left(A(\tau, \underline{\varphi}) \Phi_{\mathrm{gs}}\right)+\mathcal{O}\left(g^{2+\mu}\right)=Q_{\mathcal{T}}^{\mathrm{sup}}\left(A(\tau, \underline{\varphi}) \Phi_{\mathrm{gs}}\right)+\mathcal{O}\left(g^{2+\mu}\right) \\
& \quad=g^{2} Q_{\mathcal{T}}^{\tau}(\underline{\varphi}):=g^{2}\left(n_{1} ! n_{2} ! \cdots n_{m} !\right) \sum_{j=1}^{m} n_{j} \int_{\mathcal{T}}\left|\left\langle L_{0}^{p}, \varphi_{j}\right\rangle\right|^{2} d^{3} p
\end{aligned}
$$

Here, $L_{\infty}^{p}$ and $L_{0}^{p}$ are the distributions defined in Eqs. (I.38)-(I.40), and $\mu \equiv \mu(K):=$ $1-K^{-1}>0$, where $K$ is the degree of differentiability in Hypothesis 1 and 2. 
The asymptotic representation (I.42) (resp. (I.43)) is similar to the Born expansion for the $T$-matrix (see e.g. [22]) in Schrödinger scattering theory (resp. to the Born expansion for the wave operator).

Note that the coefficient $Q_{\mathcal{T}}^{\tau}(\underline{\varphi})$ of the leading order contribution to the photoelectric effect is additive (counting multiplicities) in the single photon states $\varphi_{1}, \ldots, \varphi_{m}$. This implies that the contribution of each $\varphi_{j}$ is independent of the other single photon states $\varphi_{i}$, $i \neq j$, in the photon cloud, in contrast to the classical model for the photoelectric effect which would have predicted a dependence of $Q_{\mathcal{T}}^{\tau}(\underline{\varphi})$ only on the total energy of the photon cloud.

\section{I.2.4 The Limit of Monochromatic Light}

For large values of $\tau$, i.e., in Case (i) in Theorem I.1 above, the transported charge directly yields Einstein's predictions (I.1) and (I.2). In Case (ii), however, there is an inconsistency between Eq. (I.43) and (I.1) and (I.2), since $\left\langle L_{0}^{p}, \varphi_{j}\right\rangle$ may be non-vanishing, even if $\varphi_{j}$ is supported in a region where $\omega(k)<p^{2}-E_{0}$. This observation may reflect that Case (ii) in Theorem I.1 is physically less relevant than Case (i), because it can hardly be realized experimentally: the photons would need to be present at the origin $x=0$ at time $t=$ 0 , coming from nowhere. On the other hand, Case (ii) deals with a (perhaps naive, but reasonable) first proposal for a model of a photon cloud, and it is appropriate to discuss this case, as well.

It turns out, that Einstein's predictions (I.1) and (I.2) can be recovered even in Case (ii), provided the incident light is sufficiently monochromatic, i.e., sharply localized in momentum space. To define our notion of monochromatic light

in precise terms, we restrict ourselves to considering a photon cloud consisting of single photon with wave function $\varphi_{\delta} \in C_{0}^{\infty}\left(\mathbb{R}^{3} \backslash\{0\}\right)$, only. It is a trivial matter to extend the consideration below to a photon cloud of $N$ orthonormal photon wave functions.

For fixed $\omega>0$, we construct a wave function $\varphi_{\delta}$ localized in energy about $\omega$ by choosing a smooth, $L^{2}(\mathbb{R})$-normalized function of one, compactly supported variable, $\chi_{1}, \chi_{2}, \ldots \chi_{m} \in C_{0}^{\infty}\left[\left(-\frac{1}{2}, \frac{1}{2}\right)\right]$, and a smooth, normalized functions on the two-sphere, $\kappa \in C^{\infty}\left[S^{2}\right]$ and setting

$$
\varphi_{\delta}\left(r, \Omega_{k}\right):=\omega^{-1} \delta^{-1 / 2} \chi\left(\frac{r-\omega_{j}}{\delta}\right) \kappa\left(\Omega_{k}\right),
$$

using spherical coordinates $\left(r, \Omega_{k}\right)=(|k|, k /|k|)$. The limit of monochromatic light is then defined to be $\delta \rightarrow 0$. Note that $\varphi_{\delta}$ is asymptotically normalized in this limit, $\lim _{\delta \rightarrow 0}\left\|\varphi_{\delta}\right\|=1$.

Given the photon wave function $\varphi_{\delta}$, a (more or less straightforward) computation in distribution theory yields, for any measurable set $\mathcal{T} \subseteq \mathbb{R}^{3}$, that the first order term of the transported charge in Case (ii) in Theorem I.1 is given by

$$
\lim _{\delta \rightarrow 0} \int_{\mathcal{T}}\left|\left\langle L_{0}^{p}, \varphi_{\delta}\right\rangle\right|^{2} d^{3} p=\left\{\begin{array}{ccc}
0 & \text { if } & E_{0}+\omega<0 \\
\Theta_{0} I_{0} & \text { if } & E_{0}+\omega=0 \\
\Theta_{0} I_{+} & \text {if } & E_{0}+\omega>0
\end{array}\right.
$$


where,

$$
\begin{aligned}
\Theta_{0} & :=\int_{\mathbb{S}^{2}} \mathbf{1}_{\mathcal{T}}\left(\sqrt{E_{0}+\omega} \sigma\right) \sqrt{E_{0}+\omega}\left|\Theta\left(\sqrt{E_{0}+\omega} \sigma, \omega\right)\right|^{2} d^{2} \sigma, \\
\Theta(p, r) & :=-i \int_{\mathbb{S}^{2}} \frac{r^{2}}{\omega} \hat{\rho}(p, r \sigma) \kappa(\sigma) d^{2} \sigma,
\end{aligned}
$$

with $\hat{\rho}(p, k)$ denoting the Fourier transform of $x \rightarrow \rho(x, k)$, and

$$
\begin{aligned}
I_{0} & :=\int_{0}^{\infty}\left|\int_{-\infty}^{\infty} \frac{\chi(x) d x}{y-x+i 0}\right|^{2} d y \\
I_{+} & :=\int_{-\infty}^{\infty}\left|\int_{-\infty}^{\infty} \frac{\chi(x) d x}{y-x+i 0}\right|^{2} d y,
\end{aligned}
$$

We state Eq. (I.45) without proof but mention that its derivation requires a slightly stronger assumption than what is provided by Hypothesis $1-2$.

Einstein's predictions (I.1) and (I.2) are now manifest in the right hand side of (I.45). The coefficient $\lim _{\delta \rightarrow 0} Q_{\mathcal{T}}^{0}\left(A_{\delta} \Phi_{\mathrm{gs}}\right)$ may still vanish, as the factor $\Theta_{0}$ may turn out to be zero. This case is in accordance with (I.1) and (I.2) and merely reflects the physical fact that some transitions (here: from a bound into a scattering state) are forbidden in lowest order perturbation theory.

\section{I.2.5 Comparison to other Results and Methods in Scattering Theory}

As pointed out in the introduction, the results of the present paper may be considered scattering theoretic, as the transported charge derives from the asymptotic limit of the unitary evolution operator $e^{-i t H_{g}}$, as $t \rightarrow \infty$. Most of the work in mathematical scattering theory in the past two decades or so is devoted to proving asymptotic completeness (AC), first for nonrelativistic $N$-body systems and, more recently and inspired by the former, for quantum field theoretic models like the one defined by the Hamiltonian $H_{g}$, especially, in $[6,7,10,11,12]$. The mathematical methods developed and applied in these papers are, perhaps, more sophisticated than those used by us for the computation of the transported charge. A central role in all the papers mentioned above is played by asymptotic observables, notably the asymptotic velocity operator. Loosely speaking, the positivity of the asymptotic velocity guarantees that any initial state eventually breaks up into parts which move independently, i.e., whose time evolution is free (noninteracting), and this is an important ingredient to prove $\mathrm{AC}$. It is therefore important to note that the transported charge measures any contribution that escapes to infinity (in particle configuration space), even those that come from particles with zero asymptotic velocity. Another important aspect to note is that we are interested in a quantitative estimate on the transported charge, asymptotically in $g$. In this context, $\mathrm{AC}$ has to be seen as an existence result: every vector can be approximated by a polynomial in asymptotic creation operators, acting on the ground states of the system. It is not easy to turn the involved estimates leading to this statement into quantitative information. Also, it should be noted that so far $\mathrm{AC}$ can be proved only for massive quantum fields - an assumption that plays no role in our analysis, although we do make a confinement assumption on the interaction couplings. 
To be more specific about the implications of $\mathrm{AC}$ on the value of the transported charge, we consider the results in [10], where $\mathrm{AC}$ is proved for Rayleigh scattering, i.e., for the energy range below the ionization threshold. In [10] an auxiliary Hilbert space $\widetilde{\mathcal{H}}=\mathcal{H} \otimes$ $\mathcal{F}$ is introduced, and with the scattering identification operator $I: \widetilde{\mathcal{H}} \longrightarrow \mathcal{H}$ given by $I\left[\varphi \otimes a^{*}\left(f_{1}\right) \cdots a^{*}\left(f_{n}\right) \Omega\right]:=a^{*}\left(f_{1}\right) \cdots a^{*}\left(f_{n}\right) \varphi$, the dynamics generated by $\widetilde{H}:=H_{g} \otimes$ $\mathbf{1}_{\mathcal{F}}+\mathbf{1}_{\mathcal{H}} \otimes H_{f}$ on $\widetilde{\mathcal{H}}$ can be compared to the dynamics on $\mathcal{H}$ generated by $H_{g}$. The main result in $[10]$ is, that

$$
\Omega_{+}:=s-\lim _{\tau \rightarrow \infty} e^{i \tau H_{g}} I e^{-i \tau \tilde{H}}\left(\mathbf{1}_{p p}\left(H_{g}\right) \otimes \mathbf{1}_{\mathcal{F}}\right)
$$

exists and $\operatorname{Ran} \Omega_{+} \supseteq \operatorname{Ran} \mathbf{1}_{\mathbb{R}_{0}^{-}}\left(H_{g}\right)$. In particular, for each $\Psi \in \operatorname{Ran} \mathbf{1}_{\mathbb{R}_{0}^{-}}\left(H_{g}\right)$, there exists $\varphi \in \tilde{\mathcal{H}}$, such that

$$
\begin{aligned}
& \limsup _{R \rightarrow \infty} \limsup _{t \rightarrow \infty}\left\|F_{R} e^{-i t H_{g}} \Psi\right\|=\limsup _{R \rightarrow \infty} \limsup _{t \rightarrow \infty}\left\|F_{R} e^{-i t H_{g}} \Omega_{+} \varphi\right\|= \\
& \quad=\limsup _{R \rightarrow \infty} \limsup _{t \rightarrow \infty}\left\|F_{R} I e^{-i t \widetilde{H}}\left(\mathbf{1}_{p p}\left(H_{g}\right) \otimes \mathbf{1}_{\mathcal{F}}\right) \varphi\right\|=0
\end{aligned}
$$

and the last equation follows from the fact that $e^{-i t \widetilde{H}}$ leaves $\mathbf{1}_{p p}(\mathcal{H})$ invariant, and the application of $I$ does not affect the electronic component, thus (B.1) implies (I.51). Hence, the proof of $\mathrm{AC}$ in [10] yields $Q^{\mathrm{inf}}=Q^{\text {sup }}=0$ below the ionization threshold. While this result does not cover the range of positive energies, which is where the photoelectric effect takes place, the proof of $\mathrm{AC}$ for Compton scattering in the more recent paper [11] may show that $Q^{\text {inf }}=Q^{\text {sup }}$ extends to any bounded energy range. It would then be interesting to see whether the methods used therein also yield a better quantitative estimate on the transported charge than the one derived in the present paper.

\section{Dyson Series and Photoelectric Effect}

In this section we study the asymptotics, as $t \rightarrow \infty$, for $e^{-i t H_{g}} A(\tau, f) \Phi_{\mathrm{gs}}$, where $H_{g}=$ $H_{0}+g W$ is the Hamiltonian generating the dynamics of the interacting system, which is applied to an initial state $A(\tau, \underline{f}) \Phi_{\mathrm{gs}}$ for $0 \leq \tau \leq g^{\mu}$ and for $\tau \geq g^{-\frac{1}{K}}$. The initial state is composed of a photon cloud

$$
A \equiv A(\underline{f})=a^{*}\left(f_{1}\right) a^{*}\left(f_{2}\right) \cdots a^{*}\left(f_{N}\right) \text {, with } f_{1}, f_{2}, \ldots, f_{N} \in C_{0}^{\infty}\left(B_{\Lambda} \backslash\{0\}\right),
$$

of $N$ photons and its time evolution

$$
A(\tau) \Phi_{\mathrm{gs}} \equiv A(\tau, \underline{f}) \Phi_{\mathrm{gs}}:=e^{-i \tau H_{g}} e^{i \tau H_{0}} A(\underline{f}) e^{-i \tau H_{0}} e^{i \tau H_{g}} \Phi_{\mathrm{gs}}
$$

applied to a ground state vector $\Phi_{\mathrm{gs}}$,

$$
H_{g} \Phi_{\mathrm{gs}}=E_{0} \Phi_{\mathrm{gs}}
$$

representing the interacting atom at rest. Note that each photon orbital $f_{j}$ is assumed to be smooth and compactly supported away from zero momentum and in the ball of radius $\Lambda<\infty$ about the origin,

$$
\operatorname{supp} f_{j} \subseteq B_{\Lambda} \backslash\{0\}:=\left\{k \in \mathbb{R}^{3} \mid 0<\omega(k)<\Lambda\right\} .
$$


We now give a list of assumptions used to derive Theorem II.1, below. Given an operator $X$ on $\mathcal{H}$ and $t \in \mathbb{R}$ we denote its free Heisenberg time evolution by

$$
X_{t}:=e^{-i t H_{0}} X e^{i t H_{0}} .
$$

Next, we recall from (I.27) the estimate

$$
\left\|\left(H_{f}^{(\Lambda)}+\mathbf{1}\right)^{\alpha} P_{c} \Phi_{\mathrm{gs}}\right\|+\left\|\left(H_{f}^{(\Lambda)}+\mathbf{1}\right)^{\alpha} P_{\Omega}^{\perp} \Phi_{\mathrm{gs}}\right\| \leq \mathcal{O}(g)
$$

for any $\alpha \geq 1$ and any $\Lambda<\infty$, where $H_{f}^{(\Lambda)}$ is defined in (I.28). Eq. (II.6) is proved in Lemma III.2, below. Furthermore, we recall from (I.22) that

$$
P_{d} W P_{d} \equiv\left(P_{d} \otimes \mathbf{1}\right) W\left(P_{d} \otimes \mathbf{1}\right)=0
$$

In Lemma III.3, we demonstrate that, for any $s \in \mathbb{R}$,

$$
\left\|\left[W, A_{s}\right]\left(H_{f}^{(\Lambda)}+\mathbf{1}\right)^{-1-(N / 2)}\right\| \leq \mathcal{O}\left((1+|s|)^{-K}\right),
$$

where $K>1$ is the degree of differentiability in Hypothesis 1 . Since $\Phi_{\mathrm{gs}} \in \operatorname{dom}\left[\left(H_{f}+\right.\right.$ 1) $\left.{ }^{1+(N / 2)}\right]$, for any $N \in \mathbb{N}_{0}$, the estimate (II.8) and the fact that $H_{f}$ and $P_{d}$ commute imply the norm-convergence of

$$
\Phi_{\infty}(A(\underline{f}), \tau):=\lim _{T \rightarrow \infty} \Phi_{T}(A(\underline{f}), \tau)
$$

where

$$
\Phi_{T}(X, \tau):= \begin{cases}\int_{0}^{T} d s e^{i s\left(H_{0}-E_{0}\right)}\left[W, X_{s}\right] P_{d} \Phi_{\mathrm{gs}} & \text { if } \quad 0 \leq \tau \leq g^{\mu} \\ \int_{-T}^{T} d s e^{i s\left(H_{0}-E_{0}\right)}\left[W, X_{s}\right] P_{d} \Phi_{\mathrm{gs}} & \text { if } \quad \tau \geq g^{-\frac{1}{K}}\end{cases}
$$

provided $K>1$. Furthermore, we show in Lemma III.4 that, for any $r \in \mathbb{R}$ and $s \in \mathbb{R}_{0}^{+}$,

$$
\left\|W e^{-i r H_{0}} P_{c}\left[W, A_{s}\right]\left(H_{f}+\mathbf{1}\right)^{-2-(N / 2)}\right\| \leq \mathcal{O}\left((1+|r|)^{-3 / 2}\right) .
$$

Under these assumptions, we prove the following Theorem.

Theorem II.1. Assume Hypotheses 1-2 and (II.1)-(II.11). Then there exists a constant $C<\infty$ such that, for all $t \geq g^{-1 / K}$,

$$
\left\|e^{-i t\left(H_{g}-E_{0}\right)} A(\tau) \Phi_{\mathrm{gs}}-A_{t} \Phi_{\mathrm{gs}}+i g e^{-i t\left(H_{0}-E_{0}\right)} \Phi_{\infty}(A, \tau)\right\| \leq C g^{1+\mu(K)},
$$

provided that either $0 \leq \tau \leq g^{\mu}$ or $\tau \geq g^{-\frac{1}{K}}$, and where $\mu(K):=1-K^{-1}>0$ and $K>1$ is the degree of differentiability in Hypothesis 1 . 
Proof. First we compute the Heisenberg time evolution of the photon cloud $A(\tau)$ on Ran $\left[\left(H_{g}-i\right)^{-1-N}\right]$,

$$
\begin{aligned}
e^{-i t H_{g}} A(\tau) e^{i t H_{g}} & =e^{-i(t+\tau) H_{g}} e^{i(t+\tau) H_{0}} A_{t} e^{-i(t+\tau) H_{0}} e^{i(t+\tau) H_{g}} \\
& =A_{t}+\left[e^{-i s H_{g}} e^{i s H_{0}} A_{t} e^{-i s H_{0}} e^{i s H_{g}}\right]_{s=0}^{s=t+\tau} \\
& =A_{t}-i g \int_{0}^{t+\tau} d s e^{-i s H_{g}}\left[W, A_{t-s}\right] e^{i s H_{g}} \\
& =A_{t}-i g \int_{-\tau}^{t} d s e^{-i(t-s) H_{g}}\left[W, A_{s}\right] e^{i(t-s) H_{g}}
\end{aligned}
$$

using $A=A(0)=A(0, \underline{f})$ and a change of integration variables, $s \mapsto t-s$. Applying (II.13) to the ground state $\bar{\Phi}_{\text {gs }}$, we obtain

$$
\begin{aligned}
-i g \Psi(t, \tau) & :=e^{-i t\left(H_{g}-E_{0}\right)} A(\tau) \Phi_{\mathrm{gs}}-A_{t} \Phi_{\mathrm{gs}} \\
& =-i g \int_{-\tau}^{t} d s e^{-i(t-s)\left(H_{g}-E_{0}\right)}\left[W, A_{s}\right] \Phi_{\mathrm{gs}}
\end{aligned}
$$

and our goal is to show that

$$
\left.\| \Psi(t, \tau)-e^{-i t\left(H_{0}-E_{0}\right)} \Phi_{\infty}(A(\tau), \tau)\right) \| \leq C g^{\mu} .
$$

To this end, we first prove (II.15) for the special case $\tau=0$. Given $T \geq 1$ and $K>1$, and assuming that $t \geq T$, we observe that

$$
\begin{gathered}
\left\|\Phi_{\infty}(A, 0)-\Phi_{T}(A, 0)\right\| \leq \mathcal{O}\left(T^{1-K}\right) \\
\left\|\Psi(t, 0)-\int_{0}^{T} d s e^{-i(t-s)\left(H_{g}-E_{0}\right)}\left[W, A_{s}\right] \Phi_{\mathrm{gs}}\right\| \leq \mathcal{O}\left(T^{1-K}\right) .
\end{gathered}
$$

Next, we use that $H_{0}$ and $A$ commute with $P_{d}$ and that $P_{d} W P_{d}=0$ which imply

$$
\left[W, A_{s}\right] P_{d}=P_{c}\left[W, A_{s}\right] P_{d} \text {. }
$$

Hence we have that

$$
\begin{aligned}
& \left\|e^{-i t H_{g}} \Phi_{T}(A, 0)-e^{-i t H_{0}} \Phi_{T}(A, 0)\right\| \\
& =g\left\|\int_{0}^{t} d r e^{-i r H_{g}} W e^{-i(t-r) H_{0}} \Phi_{T}(A, 0)\right\| \\
& =g\left\|\int_{0}^{t} d r \int_{0}^{T} d s e^{-i s E_{0}} e^{-i r H_{g}} W e^{-i(t-r-s) H_{0}} P_{c}\left[W, A_{s}\right] P_{d} \Phi_{\mathrm{gs}}\right\| \\
& \leq g\left\|\left(H_{f}+\mathbf{1}\right)^{2+(N / 2)} \Phi_{\mathrm{gs}}\right\| \\
& \quad \int_{0}^{t} d r \int_{0}^{T} d s\left\|W e^{-i(t-r-s) H_{0}} P_{c}\left[W, A_{s}\right]\left(H_{f}+\mathbf{1}\right)^{-2-(N / 2)}\right\|,
\end{aligned}
$$

and (II.11) and a change of variable $r \mapsto t-r$ now imply that

$$
\begin{aligned}
\left\|e^{-i t H_{g}} \Phi_{T}(A, 0)-e^{-i t H_{0}} \Phi_{T}(A, 0)\right\| & \leq C_{1} g \int_{0}^{T} d s \int_{0}^{t} \frac{d r}{(1+|r-s|)^{3 / 2}} \\
& \leq C_{1} C_{2} g T
\end{aligned}
$$


where $C_{1}<\infty$ and $C_{2}:=\int_{-\infty}^{\infty}(1+|r|)^{-3 / 2} d r<\infty$. Applying the estimates (II.16)(II.20) to the left side of (II.15), we hence obtain that

$$
\begin{aligned}
& \left\|\Psi(t, 0)-e^{-i t\left(H_{0}-E_{0}\right)} \Phi_{\infty}(A, 0)\right\| \\
& \leq \quad\left\|\Psi(t, 0)-\int_{0}^{T} d s e^{-i(t-s)\left(H_{g}-E_{0}\right)}\left[W, A_{s}\right] \Phi_{\mathrm{gs}}\right\| \\
& \quad+\left\|\int_{0}^{T} d s e^{-i(t-s)\left(H_{g}-E_{0}\right)}\left[W, A_{s}\right] \Phi_{\mathrm{gs}}-e^{-i t\left(H_{g}-E_{0}\right)} \Phi_{T}(A, 0)\right\| \\
& \quad+\left\|\left(e^{-i t\left(H_{g}-E_{0}\right)}-e^{-i t\left(H_{0}-E_{0}\right)}\right) \Phi_{T}(A, 0)\right\|+\left\|\Phi_{T}(A, 0)-\Phi_{\infty}(A, 0)\right\| \\
& \leq \quad\left\|\int_{0}^{T} d s e^{i s\left(H_{g}-E_{0}\right)}\left[W, A_{s}\right] \Phi_{\mathrm{gs}}-\Phi_{T}(A, 0)\right\|+\mathcal{O}\left(T^{1-K}+g T\right) \\
& \leq \quad\left\|\int_{0}^{T} d s\left(e^{i s\left(H_{g}-E_{0}\right)}-e^{i s\left(H_{0}-E_{0}\right)}\right)\left[W, A_{s}\right] P_{d} \Phi_{\mathrm{gs}}\right\| \\
& \quad+\left\|\int_{0}^{T} d s e^{i s\left(H_{g}-E_{0}\right)}\left[W, A_{s}\right] P_{c} \Phi_{\mathrm{gs}}\right\|+\mathcal{O}\left(T^{1-K}+g T\right),
\end{aligned}
$$

using that $P_{d}+P_{c}=1$. It remains to bound the two integrals in the last line of (II.21). The second of these is bounded by

$$
\begin{aligned}
& \left\|\int_{0}^{T} d s e^{i s\left(H_{g}-E_{0}\right)}\left[W, A_{s}\right] P_{c} \Phi_{\mathrm{gs}}\right\| \\
& \quad \leq\left\|\left(H_{f}^{(\Lambda)}+\mathbf{1}\right)^{1+(N / 2)} P_{c} \Phi_{\mathrm{gs}}\right\| \int_{0}^{T} d s\left\|\left[W, A_{s}\right]\left(H_{f}^{(\Lambda)}+\mathbf{1}\right)^{-1-(N / 2)}\right\| \\
& \quad \leq \mathcal{O}(g) \leq \mathcal{O}(g T),
\end{aligned}
$$

due to (II.6) and (II.8), since $K>1$ and $T \geq 1$. To estimate the first integral, we use again the DuHamel formula as in (II.19). This yields

$$
\begin{aligned}
& \left\|\int_{0}^{T} d s\left(e^{i s\left(H_{g}-E_{0}\right)}-e^{i s\left(H_{0}-E_{0}\right)}\right)\left[W, A_{s}\right] P_{d} \Phi_{\mathrm{gs}}\right\| \\
& \leq g\left\|\int_{0}^{T} d s e^{-i s E_{0}} \int_{0}^{s} d r e^{i r H_{g}} W e^{i(s-r) H_{0}} P_{c}\left[W, A_{s}\right] P_{d} \Phi_{\mathrm{gs}}\right\| \\
& \leq g\left\|\left(H_{f}+\mathbf{1}\right)^{2+(N / 2)} \Phi_{\mathrm{gs}}\right\| \\
& \quad \int_{0}^{T} d s \int_{0}^{s} d r\left\|W e^{i(s-r) H_{0}} P_{c}\left[W, A_{s}\right]\left(H_{f}+\mathbf{1}\right)^{-2-(N / 2)}\right\| \\
& \leq \quad C_{1} g \int_{0}^{T} d s \int_{0}^{s} \frac{d r}{(1+|r|)^{3 / 2}} \leq \mathcal{O}(g T)
\end{aligned}
$$

changing again the integration variable $r \mapsto s-r$. Inserting (II.22) and (II.23) into (II.21), we arrive at

$$
\left\|\Psi(t, 0)-e^{-i t\left(H_{0}-E_{0}\right)} \Phi_{\infty}(A, 0)\right\| \leq \mathcal{O}\left(T^{1-K}+g T\right)
$$

which yields (II.15) upon choosing $T:=g^{\mu(K)-1}$ and setting $\mu(K):=1-K^{-1}>0$, for the special case $\tau=0$. 
Note that if more generally $0 \leq \tau<g^{\mu}$ then $\Phi_{\infty}(A, \tau)=\Phi_{\infty}(A, 0)$ and hence

$$
\left\|A(\tau) \Phi_{\mathrm{gs}}-A \Phi_{\mathrm{gs}}\right\|=g\left\|\int_{0}^{\tau} d s e^{-i s\left(H_{g}-E_{0}\right)}\left[W, A_{-s}\right] \Phi_{\mathrm{gs}}\right\| .
$$

Additionally using (II.8), we hence conclude $\left\|A(\tau) \Phi_{\mathrm{gs}}-A \Phi_{\mathrm{gs}}\right\| \leq \mathcal{O}\left(g^{1+\mu}\right)$, proving the asymptotic expansion (II.12) in the case $\tau \in\left[0, g^{\mu}\right]$.

In order to deal with the case $\tau \in\left[g^{-1 / K}, \infty\right)$, we observe that an application of (II.25), for $t \geq g^{-\frac{1}{K}}$, in combination with the decay property (II.8) establishes

$$
\left\|e^{-i t H_{g}} A(t) \Phi_{\mathrm{gs}}-e^{-i t H_{g}} A(\tau) \Phi_{\mathrm{gs}}\right\| \leq \mathcal{O}\left(g^{1+\mu}\right),
$$

and we may replace the time-evolved state $e^{i t H_{g}} A(\tau) \Phi_{\mathrm{gs}}$ by $e^{-i t H_{g}} A(t) \Phi_{\mathrm{gs}}$ in this case. Using $\tau=t$ in Eq. (II.13), we furthermore obtain

$$
e^{-i t\left(H_{g}-E_{0}\right)} A(t) \Phi_{\mathrm{gs}}=A_{t} \Phi_{\mathrm{gs}}-i g \int_{-t}^{t} d s e^{-i(t-s) H_{g}}\left[W, A_{s}\right] e^{i(t-s) H_{g}} \Phi_{\mathrm{gs}} .
$$

Using

$$
\Psi(t, t)=\int_{-t}^{t} d s e^{-i(t-s)\left(H_{g}-E_{0}\right)}\left[W, A_{s}\right] \Phi_{\mathrm{gs}}
$$

computations running along the lines of (II.16)-(II.23) substituting $\Phi_{\infty}(A, \infty), \Phi_{T}(A, \infty)$, $\Psi(t, \infty)$ for $\Phi_{\infty}(A, 0), \Phi_{T}(A, 0), \Psi(t, 0)$ and replacing integrals of the form $\int_{0}^{T} d s$ by those of the form $\int_{-T}^{T} d s$ proves (II.12) for $\tau \geq g^{-\frac{1}{K}}$, as well.

Theorem II.1 is the main tool for the analysis of the charges transported to infinity, $Q_{\mathcal{T}}^{\inf }\left(A(\tau) \Phi_{\mathrm{gs}}\right)$ and $Q_{\mathcal{T}}^{\text {sup }}\left(A(\tau) \Phi_{\mathrm{gs}}\right)$, because it allows us to take the limits $t \rightarrow \infty$ and $R \rightarrow \infty$, as shown in the following lemma.

Lemma II.2. Assume Hypotheses 1-2, and let $A=a^{*}\left(f_{1}\right) a^{*}\left(f_{2}\right) \cdots$

$a^{*}\left(f_{N}\right)$ with $f_{1}, \ldots, f_{N} \in C_{0}^{\infty}\left(\mathbb{R}^{3} \backslash\{0\}\right)$. Then there exists a constant $C<\infty$, such that the charges transported to infinity obey

$$
Q_{\mathcal{T}}^{\sup }\left(A(\tau) \Phi_{\mathrm{gs}}\right)-C g^{2+\mu} \leq g^{2}\left\|T_{\mathcal{T}} \Phi_{\infty}(A, \tau)\right\|^{2} \leq Q_{\mathcal{T}}^{\inf }\left(A(\tau) \Phi_{\mathrm{gs}}\right)+C g^{2+\mu}
$$

for $0 \leq \tau \leq g^{\mu}$ and $\tau \geq g^{-\frac{1}{K}}$, where $\mu(K):=1-K^{-1}>0$ and $K>1$ is the degree of differentiability in Hypothesis 1.

Proof: First we observe that, since $F_{R} \equiv F_{R} \otimes \mathbf{1}_{f}$ commutes with $A_{t} \equiv \mathbf{1}_{e l} \otimes A_{t}$ and $H_{f}=\mathbf{1}_{e l} \otimes H_{f}$, we have

$$
\begin{aligned}
\left\|T_{\mathcal{T}} F_{R} A_{t} \Phi_{\mathrm{gs}}\right\| & \leq\left\|F_{R} A_{t} \Phi_{\mathrm{gs}}\right\|=\left\|A_{t} F_{R} \Phi_{\mathrm{gs}}\right\| \\
& \leq\left\|A_{t}\left(H_{f}+1\right)^{-N / 2}\right\| \cdot\left\|F_{R}\left(H_{f}+1\right)^{N / 2} \Phi_{\mathrm{gs}}\right\| .
\end{aligned}
$$


Applying Lemma III.1 $N$-times, we observe that $\left\|A_{t}\left(H_{f}+1\right)^{-N / 2}\right\|$ is bounded by $C_{2}<$ $\infty$, uniformly in $t$,

$$
\left\|A_{t}\left(H_{f}+1\right)^{-N / 2}\right\| \leq \prod_{j=1}^{N}\left\|\left(H_{f}+1\right)^{(j-1) / 2} a^{*}\left(e^{-i t \omega} f_{j}\right)\left(H_{f}+1\right)^{-j / 2}\right\| \leq C_{2} .
$$

Moreover, $\left(H_{f}+1\right)^{N / 2} \Phi_{\mathrm{gs}} \in \mathcal{H}$ and $F_{R} \rightarrow 0$ strongly, as $R \rightarrow \infty$, hence

$$
\lim _{R \rightarrow \infty} \lim _{t \rightarrow \infty}\left\|T_{\mathcal{T}} F_{R} A_{t} \Phi_{\mathrm{gs}}\right\|=0 .
$$

According to Theorem II.1 we have

$$
e^{-i t\left(H_{g}-E_{0}\right)} A(\tau) \Phi_{\mathrm{gs}}=A_{t} \Phi_{\mathrm{gs}}-i g e^{-i t\left(H_{0}-E_{0}\right)} \Phi_{\infty}(A, \tau)+\operatorname{Rem}(t),
$$

where $\|\operatorname{Rem}(t)\| \leq C_{1} g^{1+\mu}$, for some constant $C_{1}<\infty$ independent of $t \in \mathbb{R}^{+}$and $\mu=1-1 / K$. Inserting Eqs. (II.33) and (II.32) into the definitions (I.31)-(I.32) of the transported charges, we find that

$$
Q_{\mathcal{T}}^{\text {sup }}\left(A(\tau) \Phi_{\mathrm{gs}}\right)-C_{1} g^{2+\mu} \leq g^{2} \widetilde{Q}_{\mathcal{T}}\left(A(\tau) \Phi_{\mathrm{gs}}\right) \leq Q_{\mathcal{T}}^{\mathrm{inf}}\left(A(\tau) \Phi_{\mathrm{gs}}\right)+C_{1} g^{2+\mu},
$$

where

$$
\left.\widetilde{Q}_{\mathcal{T}}\left(A(\tau) \Phi_{\mathrm{gs}}\right):=\lim _{R \rightarrow \infty} \lim _{t \rightarrow \infty} \| T_{\mathcal{T}} F_{R} e^{-i t\left(H_{0}-E_{0}\right)} \Phi_{\infty}(A, \tau)\right) \|^{2},
$$

and estimate (II.29) would follow from $\widetilde{Q}_{\mathcal{T}}\left(A(\tau) \Phi_{\mathrm{gs}}\right)=\left\|T_{\mathcal{T}} \Phi_{\infty}(A, \tau)\right\|^{2}$.

Next, we turn to $T_{\mathcal{T}} F_{R} e^{-i t\left(H_{0}-E_{0}\right)} \Phi_{\infty}(A, \tau)$. We note that $F_{R}=F_{R} P_{c}$ and hence

$$
F_{R} e^{-i t\left(H_{0}-E_{0}\right)}=e^{-i t\left(H_{f}-E_{0}\right)} F_{R} P_{c} e^{-i t H_{e l}}=e^{-i t\left(H_{f}-E_{0}\right)} F_{R} e^{-i t(-\Delta)} P_{c} \text {. }
$$

Furthermore, the absolute continuity of the spectrum of $-\Delta$ on $L^{2}\left(\mathbb{R}^{3}\right)$ implies that

$$
\left(1-F_{R}\right) e^{-i t(-\Delta)}=\mathbf{1}_{\{|x|<R\}} e^{-i t(-\Delta)} \rightarrow 0
$$

strongly, as $t \rightarrow \infty$, for any $R<\infty$. Using (II.36), (II.37), and the fact that the operators $T_{\mathcal{T}}=T_{\mathcal{T}} P_{c}, e^{-i t(-\Delta)}$, and $e^{-i t\left(H_{f}-E_{0}\right)}$ commute, we thus obtain

$$
\begin{aligned}
& \lim _{R \rightarrow \infty} \lim _{t \rightarrow \infty}\left\|T_{\mathcal{T}} F_{R} e^{-i t\left(H_{0}-E_{0}\right)} \Phi_{\infty}(A, \tau)\right\|= \\
& \quad=\lim _{R \rightarrow \infty} \lim _{t \rightarrow \infty}\left\|T_{\mathcal{T}} F_{R} e^{-i t(-\Delta)} P_{c} \Phi_{\infty}(A, \tau)\right\|=\left\|T_{\mathcal{T}} \Phi_{\infty}(A, \tau)\right\|,
\end{aligned}
$$

which implies the claim.

Next, we recall from Eq. (II.9) the definition of

$$
\Phi_{\infty}(A, \tau)= \begin{cases}\int_{0}^{\infty} d s e^{i s\left(H_{0}-E_{0}\right)}\left[W, A_{s}\right] P_{d} \Phi_{\mathrm{gs}} & \text { if } 0 \leq \tau \leq g^{\mu} \\ \int_{-\infty}^{\infty} d s e^{i s\left(H_{0}-E_{0}\right)}\left[W, A_{s}\right] P_{d} \Phi_{\mathrm{gs}} & \text { if } \tau \geq g^{-\frac{1}{K}}\end{cases}
$$


which we complement by the definition of the vector

$$
\Phi_{\infty}^{\Omega}(A, \tau):= \begin{cases}\int_{0}^{\infty} d s e^{i s\left(H_{0}-E_{0}\right)}\left[W, A_{s}\right] P_{d}(0 \oplus \Omega) & \text { if } 0 \leq \tau \leq g^{\mu} \\ \int_{-\infty}^{\infty} d s e^{i s\left(H_{0}-E_{0}\right)}\left[W, A_{s}\right] P_{d}(0 \oplus \Omega) & \text { if } \tau \geq g^{-\frac{1}{K}}\end{cases}
$$

whose existence is guaranteed thanks to Lemma III.3. Lemma II.3 below shows that these two vectors differ by at most $\mathcal{O}(g)$, i.e., a replacement of the ground state $\Phi_{\text {gs }}$ of $H_{g}$ by the ground state $0 \oplus \Omega$ of $H_{0}$ in the definition of $\Phi_{\infty}(A, \tau)$ introduces only small errors which are negligible, as we shall see.

Lemma II.3. Assume Hypotheses 1-2, and let $A=a^{*}\left(f_{1}\right) a^{*}\left(f_{2}\right) \cdots a^{*}\left(f_{N}\right)$ with $f_{1}, \ldots$, $f_{N} \in C_{0}^{\infty}\left(\mathbb{R}^{3} \backslash\{0\}\right)$. Then there exists a constant $C<\infty$, such that

$$
\left\|\Phi_{\infty}(A, \tau)-\Phi_{\infty}^{\Omega}(A, \tau)\right\| \leq C g
$$

Proof: We first note that due to Lemma III.3, we have the estimate

$$
\begin{aligned}
& \left\|\Phi_{\infty}(A, \tau)-\Phi_{\infty}^{\Omega}(A, \tau)\right\| \leq \\
& \quad \leq\left\|\left(H_{f}^{(\Lambda)}+1\right)^{1+\frac{N}{2}}\left(P_{d} \Phi_{\mathrm{gs}}-(0 \oplus \Omega)\right)\right\| \cdot \int_{\mathbb{R}} d s\left\|\left[W, A_{s}\right]\left(H_{f}^{(\Lambda)}+1\right)^{-1-\frac{N}{2}}\right\| \\
& \quad \leq C_{1}\left\|\left(H_{f}^{(\Lambda)}+1\right)^{1+\frac{N}{2}}\left(P_{d} \Phi_{\mathrm{gs}}-(0 \oplus \Omega)\right)\right\|
\end{aligned}
$$

for some constant $C_{1}<\infty$. Additionally using Lemma III.2 and the decomposition $P_{d} \Phi_{\mathrm{gs}}-(0 \oplus \Omega)=\left[P_{d} P_{\Omega} \Phi_{\mathrm{gs}}-(0 \oplus \Omega)\right]+P_{d} P_{\Omega}^{\perp} \Phi_{\mathrm{gs}}$, we observe that

$$
\left\|\left(H_{f}^{(\Lambda)}+1\right)^{1+N / 2}\left(P_{d} \Phi_{\mathrm{gs}}-(0 \oplus \Omega)\right)\right\| \leq \mathcal{O}(g) .
$$

The final ingredient for the proof of Theorem I.1 is Lemma II.4, below. Note that due to the definitions (II.40) we may restrict ourselves to the case $\tau=0$ and $\tau=\infty$.

Lemma II.4. Assume Hypotheses $1-2$, fix $N, m \in \mathbb{N}$ with $m \leq N$, and let $\varphi_{1}, \varphi_{2}, \ldots \varphi_{m} \in$ $C_{0}^{\infty}\left(\mathbb{R}^{3} \backslash\{0\}\right)$ be an orthonormal system, $\left\langle\varphi_{i} \mid \varphi_{j}\right\rangle=\delta_{i, j}$. Let $A=a^{*}\left(\varphi_{1}\right)^{n_{1}} a^{*}\left(\varphi_{2}\right)^{n_{2}} \ldots$ $a^{*}\left(\varphi_{m}\right)^{n_{m}}$, where $n_{j} \in \mathbb{N}$ are such that $n_{1}+n_{2}+\ldots+n_{m}=N$, and fix a measurable set $\mathcal{T} \subseteq \mathbb{R}^{3}$. Then

$$
\begin{aligned}
\left\|T_{\mathcal{T}} \Phi_{\infty}^{\Omega}(A, \tau)\right\|^{2} & =Q_{\mathcal{T}}^{\tau}(\underline{\varphi})= \\
& =\left(n_{1} ! n_{2} ! \cdots n_{m} !\right) \sum_{j=1}^{m} n_{j} \int_{\mathcal{T}}\left|\left\langle L_{\tau}^{p}, \varphi_{j}\right\rangle\right|^{2} d^{3} p
\end{aligned}
$$

with $L_{\tau}^{p}=L_{0}^{p}$, for $\tau \in\left[0, g^{\mu}\right]$, and $L_{\tau}^{p}=L_{\infty}^{p}$, for $\tau \in\left[g^{-1 / K}, \infty\right)$, where $L_{0}^{p}$ and $L_{\infty}^{p}$ are defined in Eqs. (I.38)-(I.40). 
Proof: We first write $W=a^{*}(G)+a(G)$ and use the canonical commutation relations to obtain that

$$
\begin{aligned}
{\left[W, A_{s}\right] } & =\left[a^{*}(G)+a(G), a^{*}\left(e^{-i s \omega} \varphi_{1}\right)^{n_{1}} a^{*}\left(e^{-i s \omega} \varphi_{2}\right)^{n_{2}} \cdots a^{*}\left(e^{-i s \omega} \varphi_{m}\right)^{n_{m}}\right] \\
& =\left[a(G), a^{*}\left(e^{-i s \omega} \varphi_{1}\right)^{n_{1}} a^{*}\left(e^{-i s \omega} \varphi_{2}\right)^{n_{2}} \cdots a^{*}\left(e^{-i s \omega} \varphi_{m}\right)^{n_{m}}\right] \\
& =\sum_{j=1}^{m} n_{j}\left\{\left\langle G \mid e^{-i s \omega} \varphi_{j}\right\rangle \otimes \prod_{i=1}^{m} a^{*}\left(e^{-i s \omega} \varphi_{i}\right)^{n_{i}-\delta_{i, j}}\right\}
\end{aligned}
$$

on $\operatorname{dom}\left[H_{f}^{1+N / 2}\right]$, where $\left\langle G \mid e^{-i s \omega} \varphi_{j}\right\rangle \in \mathcal{B}\left(\mathcal{H}_{e l}\right)$ is a bounded operator acting on the electron variables given by

$$
\left\langle G \mid e^{-i s \omega} \varphi_{j}\right\rangle:=\int d^{3} k e^{-i s \omega(k)} \varphi_{j}(k) G^{*}(k) .
$$

Moreover, for any $s \geq 0$,

$$
\begin{aligned}
& e^{i s\left(H_{0}-E_{0}\right)}\left\langle G \mid e^{-i s \omega} \varphi_{j}\right\rangle \otimes \prod_{i=1}^{m} a^{*}\left(e^{-i s \omega} \varphi_{i}\right)^{n_{i}-\delta_{i, j}}(0 \oplus \Omega) \\
& \quad=e^{i s\left(H_{e l}-E_{0}\right)}\left\langle G \mid e^{-i s \omega} \varphi_{j}\right\rangle \otimes e^{i s H_{f}} \prod_{i=1}^{m} a^{*}\left(e^{-i s \omega} \varphi_{i}\right)^{n_{i}-\delta_{i, j}}(0 \oplus \Omega) \\
& =e^{i s\left(-\Delta-E_{0}\right)}\left\langle P_{c} G P_{d} \mid e^{-i s \omega} \varphi_{j}\right\rangle \otimes \prod_{i=1}^{m} a^{*}\left(\varphi_{i}\right)^{n_{i}-\delta_{i, j}}(0 \oplus \Omega) \\
& =\left(\psi_{j, s} \oplus 0\right) \otimes \prod_{i=1}^{m} a^{*}\left(\varphi_{i}\right)^{n_{i}-\delta_{i, j}} \Omega
\end{aligned}
$$

where the Fourier transform of $\psi_{j} \in L^{2}\left(\mathbb{R}^{3}\right)$ with respect to the particle coordinate $x$ is given by

$$
\hat{\psi}_{j, s}(p)=\int d^{3} k e^{i s\left(p^{2}-E_{0}-\omega(k)\right)} \hat{\rho}(p, k) \varphi_{j}(k) .
$$

Here, we used the fact that $P_{d} G(k)^{*} P_{d}=0$ and hence $G(k)^{*} P_{d}=P_{c} G(k)^{*} P_{d}$, for all $k \in \mathbb{R}^{3}$. Moreover, $P_{c} G(k)^{*} P_{d}(\tilde{\psi} \oplus z)=z p_{\downarrow}(k)^{*} \oplus 0$, and this yields (II.48). Eqs. (II.45)(II.48) imply that

$$
\Phi_{\infty}^{\Omega}(A, \tau)=\sum_{j=1}^{m} n_{j}\left\{\int_{-\tau}^{\infty} d s\left(\psi_{j, s} \oplus 0\right)\right\} \otimes \prod_{i=1}^{m} a^{*}\left(\varphi_{i}\right)^{n_{i}-\delta_{i, j}} \Omega
$$

Passing to the momentum representation for the particle variable and using that

$$
\left\langle\prod_{i=1}^{m} a^{*}\left(\varphi_{i}\right)^{n_{i}-\delta_{i, j}} \Omega \mid \prod_{i=1}^{m} a^{*}\left(\varphi_{i}\right)^{n_{i}-\delta_{i, \ell}} \Omega\right\rangle=\delta_{j, \ell} \cdot \prod_{i=1}^{m}\left(n_{i}-\delta_{i, j}\right) !
$$

we hence obtain

$$
\begin{aligned}
\left\|T_{\mathcal{T}} \Phi_{\infty}^{\Omega}(A, \tau)\right\|^{2} & =\left\|\sum_{j=1}^{m} n_{j}\left\{\int_{-\tau}^{\infty} d s\left(T_{\mathcal{T}} \psi_{j, s} \oplus 0\right)\right\} \otimes \prod_{i=1}^{m} a^{*}\left(\varphi_{i}\right)^{n_{i}-\delta_{i, j}} \Omega\right\|^{2} \\
& =n_{1} ! n_{2} ! \cdots n_{m} ! \sum_{j=1}^{m} n_{j} \int_{\mathcal{T}} d^{3} p\left|\int_{-\tau}^{\infty} d s \hat{\psi}_{j, s}(p)\right|^{2}
\end{aligned}
$$


It remains to evaluate the integral $\int_{-\tau}^{\infty} d s \hat{\psi}_{j, s}(p)$ in (II.51), which is an exercise in distribution theory. To this end, we note that the $K>1$-fold partial differentiability of $\rho(x, k)$ with respect to $k$ in Hypothesis 1 implies that $\hat{\rho}(p, \cdot) \varphi_{j}$ is two times partially differentiable with respect to $k$, and that its derivatives of order $\leq 2$ have bounded support away from $k=0$. Since the phase in $\hat{\psi}_{j, s}(p)$ is non-stationary, away from $k=0$, two times integration by parts back and forth yields

$$
\begin{aligned}
& \int_{1}^{\infty} d s \hat{\psi}_{j, s}(p)=\int_{1}^{\infty} d s \int d^{3} k e^{i s\left(p^{2}-E_{0}-\omega(k)\right)} \hat{\rho}(p, k) \varphi_{j}(k) \\
& =\int_{1}^{\infty} \frac{d s}{s^{2}} \int d^{3} k e^{i s\left(p^{2}-E_{0}-\omega(k)\right)}\left(i \nabla \cdot \frac{k}{|k|}\right)^{2}\left[\hat{\rho}(p, k) \varphi_{j}(k)\right] \\
& =\lim _{\varepsilon \rightarrow 0} \int_{1}^{\infty} \frac{d s}{s^{2}} \int d^{3} k e^{i s\left(p^{2}-E_{0}-\omega(k)+i \varepsilon\right)}\left(i \nabla \cdot \frac{k}{|k|}\right)^{2}\left[\hat{\rho}(p, k) \varphi_{j}(k)\right] \\
& =\lim _{\varepsilon \rightarrow 0} \int d^{3} k \int_{1}^{\infty} d s e^{i s\left(p^{2}-E_{0}-\omega(k)+i \varepsilon\right)} \hat{\rho}(p, k) \varphi_{j}(k) \\
& =\lim _{\varepsilon \rightarrow 0} \int d^{3} k \frac{-i e^{i\left(p^{2}-E_{0}-\omega(k)\right)} \hat{\rho}(p, k)}{p^{2}-E_{0}-\omega(k)+i \varepsilon} \varphi_{j}(k)
\end{aligned}
$$

Thus in the case $\tau=0$ :

$$
\begin{aligned}
\int_{0}^{\infty} d s \hat{\psi}_{j, s}(p) & =\int_{0}^{1} d s \hat{\psi}_{j, s}(p)+\int_{1}^{\infty} d s \hat{\psi}_{j, s}(p) \\
& =\lim _{\varepsilon \rightarrow 0} \int d^{3} k \frac{-i \hat{\rho}(p, k)}{p^{2}-E_{0}-\omega(k)+i \varepsilon} \varphi_{j}(k)=\left\langle L_{0}^{p}, \varphi_{j}\right\rangle .
\end{aligned}
$$

For the case $\tau=\infty$, we get

$$
\begin{aligned}
\lim _{t \rightarrow \infty} \int_{-t}^{t} d s \hat{\psi}_{j, s}(p) & =\lim _{t \rightarrow \infty} \int_{-t}^{t} d s \int d^{3} k e^{i s\left(p^{2}-E_{0}-\omega(k)\right)} \hat{\rho}(p, k) \varphi_{j}(k)=\quad \text { (II.54) } \\
& =\lim _{t \rightarrow \infty} \int d^{3} k \frac{e^{i t\left(p^{2}-E_{0}-\omega(k)\right)}-e^{i t\left(p^{2}-E_{0}-\omega(k)\right)}}{i\left(p^{2}-E_{0}-\omega(k)\right)} \hat{\rho}(p, k) \varphi_{j}(k) \\
& =\lim _{t \rightarrow \infty} 2 \int d^{3} k \frac{\sin \left(t\left(p^{2}-E_{0}-\omega(k)\right)\right)}{p^{2}-E_{0}-\omega(k)} \hat{\rho}(p, k) \varphi_{j}(k)= \\
& =2 \pi \int_{\mathbb{S}^{2}} d^{2} \Omega\left(p^{2}-E_{0}\right)^{2} \hat{\rho}\left(p ; p^{2}-E_{0}, \Omega\right) \varphi_{j}\left(p^{2}-E_{0}, \Omega\right) .
\end{aligned}
$$

Proof of Theorem I.1: The proof of Theorem I.1 consists of the following chain of estimates,

$$
\begin{aligned}
Q_{\mathcal{T}}^{\text {inf }}\left(A(\tau) \Phi_{\mathrm{gs}}\right)+\mathcal{O}\left(g^{2+\mu}\right) & =Q_{\mathcal{T}}^{\text {sup }}\left(A(\tau) \Phi_{\mathrm{gs}}\right)+\mathcal{O}\left(g^{2+\mu}\right) \\
& =g^{2}\left\|T_{\mathcal{T}} \Phi_{\infty}(A, \tau)\right\|^{2} \\
& =g^{2}\left\|T_{\mathcal{T}} \Phi_{\infty}^{\Omega}(A, \tau)\right\|^{2}+\mathcal{O}\left(g^{2+\mu}\right) \\
& =Q_{\mathcal{T}}^{\tau}(\underline{\varphi})+\mathcal{O}\left(g^{2+\mu}\right),
\end{aligned}
$$

where Lemma II.2, II.3, and II.4 justify Eq. (II.55), (II.56), and (II.57), respectively. 


\section{Technical Estimates}

In this section we derive the estimates (II.6), (II.8), and (II.11), which is the basic input for the asymptotics of the time evolution asserted in Theorem II.1.

Before we turn to these bounds, we derive a preparatory lemma.

Lemma III.1. Suppose that $\alpha \geq 1, \Lambda<\infty$, and let $G \in L^{2}\left[\mathbb{R}^{3} ; \mathcal{B}(\mathcal{H})\right]$ with $C:=$ $\int d^{3} k\left(1+\omega(k)^{-1}\right)\|G(k)\|^{2}<\infty$. Denote $a^{*}(G):=\int d^{3} k G(k) \otimes a^{*}(k)$ and $a(G):=$ $\int d^{3} k G(k)^{*} \otimes a(k)$. Then

$$
\begin{aligned}
\left\|\left(H_{f}^{(\Lambda)}+1\right)^{\alpha} a(G)\left(H_{f}+1\right)^{-\alpha-1 / 2}\right\| & \leq \sqrt{C} \\
\left\|\left(H_{f}^{(\Lambda)}+1\right)^{\alpha} a^{*}(G)\left(H_{f}+1\right)^{-\alpha-1 / 2}\right\| & \leq \sqrt{C}(2+4 \Lambda)^{\alpha}, \\
\left\|\left(H_{f}^{(\Lambda)}+1\right)^{\alpha} a\left(G_{\Lambda}\right)\left(H_{f}^{(\Lambda)}+1\right)^{-\alpha-1 / 2}\right\| & \leq \sqrt{C} \\
\left\|\left(H_{f}^{(\Lambda)}+1\right)^{\alpha} a^{*}\left(G_{\Lambda}\right)\left(H_{f}^{(\Lambda)}+1\right)^{-\alpha-1 / 2}\right\| & \leq \sqrt{C}(2+4 \Lambda)^{\alpha},
\end{aligned}
$$

where $G_{\Lambda}(k):=\mathbf{1}_{\{\omega(k)<\Lambda\}} G(k)$, and $\mathbf{1}_{\{\omega(k)<\Lambda\}}$ denotes the characteristic function of $\left\{k \in \mathbb{R}^{3} \mid \omega(k)<\Lambda\right\}$.

Proof. First, we introduce a more compact notation,

$$
\begin{array}{llll}
G_{1}:=G, & H_{1}:=H_{f}, & \omega_{1}:=\omega \\
G_{2}:=G_{\Lambda}, & H_{2}:=H_{f}^{(\Lambda)}, & \omega_{2}:=\omega_{\Lambda}
\end{array}
$$

and observe that Eqs. (III.1)-(III.4) are equivalent to

$$
\begin{aligned}
\left\|\left(H_{f}^{(\Lambda)}+1\right)^{\alpha} a\left(G_{j}\right)\left(H_{j}+1\right)^{-\alpha-1 / 2}\right\| & \leq \sqrt{C} \\
\left\|\left(H_{f}^{(\Lambda)}+1\right)^{\alpha} a^{*}\left(G_{j}\right)\left(H_{j}+1\right)^{-\alpha-1 / 2}\right\| & \leq \sqrt{C}(2+4 \Lambda)^{\alpha},
\end{aligned}
$$

with $j=1,2$. We apply the operator on the left side of (III.6) to a normalized vector $\psi \in \mathcal{H}$ and obtain the desired estimate by means of the pull-through formula, the Cauchy-Schwarz inequality, and $H_{f}^{(\Lambda)}+1 \leq H_{j}+1+\omega_{j}$,

$$
\begin{aligned}
& \left\|\left(H_{f}^{(\Lambda)}+1\right)^{\alpha} a\left(G_{j}\right)\left(H_{j}+1\right)^{-\alpha-1 / 2} \psi\right\| \\
& \leq \int d^{3} k\left\|G_{j}(k)\right\|\left\|\frac{H_{f}^{(\Lambda)}+1}{H_{j}+1+\omega_{j}(k)}\right\|^{\alpha}\left\|a(k)\left(H_{j}+1\right)^{-1 / 2} \psi\right\| \\
& \leq\left(\int \frac{\left\|G_{j}(k)\right\|^{2} d^{3} k}{\omega_{j}(k)}\right)^{1 / 2} \cdot\left\|\frac{H_{j}}{H_{j}+1}\right\|^{1 / 2} \leq\left(\int \frac{\left\|G_{j}(k)\right\|^{2} d^{3} k}{\omega_{j}(k)}\right)^{1 / 2}
\end{aligned}
$$


for any $\psi \in \mathcal{H},\|\psi\|=1$. To derive (III.7), we use the canonical commutation relations,

$$
\begin{aligned}
\left\|\left(H_{f}^{(\Lambda)}+1\right)^{\alpha} a^{*}\left(G_{j}\right)\left(H_{j}+1\right)^{-\alpha-1 / 2} \psi\right\|^{2} & \\
= & \int d^{3} k d^{3} \tilde{k}\langle\psi| G_{j}(k)^{*} G_{j}(\tilde{k}) \otimes\left(H_{j}+1\right)^{-\alpha-1 / 2} a(k)\left(H_{f}^{(\Lambda)}+1\right)^{2 \alpha} a^{*}(\tilde{k}) \\
= & \int d^{3} k d^{3} \tilde{k}\left\langle a(\tilde{k})\left(H_{j}+1\right)^{-1 / 2} \psi\right| G_{j}(k)^{*} G_{j}(\tilde{k}) \\
& +\int d^{3} k\left\langle\psi \mid G_{j}(k)^{*} G_{j}(k) \otimes\left(H_{j}+1\right)^{-2 \alpha-1}\left(H_{f}^{(\Lambda)}+1+\omega_{\Lambda}(k)\right)^{2 \alpha} \psi\right\rangle \\
\leq & \left.\| \frac{H_{f}^{(\Lambda)}+1+2 \Lambda}{\left(H_{j}^{(\Lambda)}+1+\omega_{\Lambda}(k)+\omega_{\Lambda}(\tilde{k})\right)^{2}}\right]^{\alpha}\left(\int d^{3} k\left\|G_{j}(k)\right\|\left\|a(k)\left(H_{j}+1\right)^{-1 / 2} \psi\right\|\right)^{2} \\
& +\left\|\left(H_{j}+1\right)^{-2 \alpha-1}\left(H_{f}^{(\Lambda)}+1+\omega_{\Lambda}(k)\right)^{2 \alpha}\right\|\left(\int d^{3} k\left\|G_{j}(k)\right\|^{2}\right) \\
\leq & C(1+2 \Lambda)^{2 \alpha}\left(1+\int d^{3} k \omega_{j}(k)\left\|a(k)\left(H_{j}+1\right)^{-1 / 2} \psi\right\|^{2}\right) \\
\leq & C(1+2 \Lambda)^{2 \alpha}\left(1+\left\|\frac{H_{j}}{H_{j}+1}\right\|\right) \leq C(2+4 \Lambda)^{2 \alpha} .
\end{aligned}
$$

Note that in the last step we used $H_{j}=\int d^{3} k \omega_{j}(k) a^{*}(k) a(k)$.

Now we are in position to establish Eq. (II.6) which shows that the interacting atomphoton ground state $\Phi_{g s}$ is well-localized in energy, even for the noninteracting Hamiltonian.

Lemma III.2. For any $\alpha \geq 1, \Lambda<\infty$, and sufficiently small $g>0$, we have

$$
\left\|\left(H_{f}^{(\Lambda)}+\mathbf{1}\right)^{\alpha} P_{c} \Phi_{\mathrm{gs}}\right\|+\left\|\left(H_{f}^{(\Lambda)}+\mathbf{1}\right)^{\alpha} P_{\Omega}^{\perp} \Phi_{\mathrm{gs}}\right\| \leq \mathcal{O}(g) .
$$

Proof. The proof is similar to the one for [2, Thm. X]. We first note that the asserted bound (III.10) is implied by (I.25) and the following estimate,

$$
\left\|\left(H_{f}^{(\Lambda)}+\mathbf{1}\right)^{\alpha} \mathbf{1}_{\left\{H_{0} \geq E_{0} / 3\right\}} \Phi_{\mathrm{gs}}\right\| \leq \mathcal{O}(g),
$$

because on $\operatorname{Ran} 1_{\left\{H_{0}<E_{0} / 3\right\}}$, we have $P_{c}=0$ and $H_{f}^{(\Lambda)}<\left|e_{0}\right|-\left|E_{0}\right| / 3$.

Let $\chi \in C_{0}^{\infty}(I ;[0,1])$ be a real-valued, smooth function, compactly supported in $I:=$ $\left(\frac{4}{3} E_{0}, \frac{2}{3} E_{0}\right)$ and such that $\chi\left(E_{0}\right)=1$. Clearly, $\mathbf{1}_{\left\{H_{0} \geq E_{0} / 3\right\}} \chi\left(H_{0}\right)=0$, and $\chi\left(H_{g}\right) \Phi_{\mathrm{gs}}=$ $\Phi_{\mathrm{gs}}$. Hence we observe that

$$
\begin{aligned}
& \left(H_{f}^{(\Lambda)}+1\right)^{\alpha} \mathbf{1}_{\left\{H_{0} \geq E_{0} / 3\right\}} \Phi_{\mathrm{gs}}= \\
& \mathbf{1}_{\left\{H_{0} \geq E_{0} / 3\right\}}\left(H_{f}^{(\Lambda)}+1\right)^{\alpha}\left[\chi\left(H_{g}\right)-\chi\left(H_{0}\right)\right]\left(H_{g}-E_{0}+1\right)^{-\alpha-1} \Phi_{\mathrm{gs}}
\end{aligned}
$$


Next, we represent $\chi\left(H_{g}\right)$ and $\chi\left(H_{0}\right)$ by the functional calculus based on almost analytic extensions of smooth functions of compact support [19]. Let $\tilde{\chi} \in C_{0}^{\infty}(\Delta ; \mathbb{C})$ be an almost analytic extension of $\chi$, supported in a small complex neighborhood $\Delta \subseteq \mathbb{C}$ of $I$ and such that $\bar{\partial} \tilde{\chi}(z)=\mathcal{O}\left((\operatorname{Im} z)^{2}\right)$. By means of the measure $d \mu(z):=(2 \pi i)^{-1} \overline{\bar{\partial}} \tilde{\chi}(z) d z \wedge d \bar{z}$ and the second resolvent equation, we have the following identity

$$
\begin{aligned}
\chi\left(H_{g}\right)-\chi\left(H_{0}\right) & =\int \frac{d \mu(z)}{H_{g}-z}-\frac{d \mu(z)}{H_{0}-z} \\
& =-g \int d \mu(z)\left\{\left(H_{0}-z\right)^{-1} W\left(H_{g}-z\right)^{-1}\right\}
\end{aligned}
$$

Inserting this identity into (III.12) and applying Lemma III.1 to $W=a^{*}(G)+a(G)$, we obtain the norm estimate

$$
\begin{aligned}
& \left\|\left(H_{f}^{(\Lambda)}+1\right)^{\alpha} \mathbf{1}_{\left\{H_{0} \geq E_{0} / 3\right\}} \Phi_{\mathrm{gs}}\right\| \\
& \leq g \| \int d \mu(z)\left\{\left(H_{0}-z\right)^{-1}\left(H_{f}^{(\Lambda)}+1\right)^{\alpha} W\right. \\
& \left.\cdot\left(H_{g}-E_{0}+1\right)^{-\alpha-1}\left(H_{g}-z\right)^{-1}\right\} \Phi_{\mathrm{gs}} \| \\
& \leq g \int|d \mu(z)|\left\{\left\|\left(H_{0}-z\right)^{-1}\right\|\left\|\left(H_{f}^{(\Lambda)}+1\right)^{\alpha} W\left(H_{f}+1\right)^{-\alpha-1}\right\|\right. \\
& \left.\quad \cdot\left\|\left(H_{f}+1\right)^{\alpha+1}\left(H_{g}-E_{0}+1\right)^{-\alpha-1}\right\|\left\|\left(H_{g}-z\right)^{-1}\right\|\right\}
\end{aligned}
$$

which finishes the proof.

Our next goal is the derivation of (II.8). Actually, we prove a somewhat stronger estimate which is also used in the proof of Lemma III.4, below. Recall that $A \equiv A(f)=$ $a^{*}\left(f_{1}\right) a^{*}\left(f_{2}\right) \cdots a^{*}\left(f_{N}\right)$ is a photon cloud of $N$ photons $f_{1}, f_{2}, \ldots, f_{N} \in C_{0}^{\infty}\left(B_{\Lambda} \backslash\{0\}\right)$, that $X_{t}:=e^{-i t H_{0}} X e^{i t H_{0}}$ denotes the free time evolution of an observable $X$, and that $K>1$ is the degree of differentiability in Hypothesis 1 . Denoting

$$
(1+|x|)^{3 / 2}:=\left(\begin{array}{cc}
(1+|x|)^{3 / 2} & 0 \\
0 & 1
\end{array}\right) \otimes \mathbf{1}
$$

we prove the following lemma.

Lemma III.3. For any $N \in \mathbb{N}$ and $K>1$, there exists a constant $C<\infty$ such that

$$
\left\|(1+|x|)^{3 / 2}\left[W, A_{s}\right]\left(H_{f}^{(\Lambda)}+\mathbf{1}\right)^{-1-(N / 2)}\right\| \leq C(1+|s|)^{-K},
$$

for all $s \in \mathbb{R}$

Proof. First observe that on $\operatorname{dom}\left(H_{f}^{N / 2}\right)$, we have $e^{-i s H_{0}} a^{*}(f) e^{i s H_{0}}=a^{*}\left(e^{-i s \omega} f\right)$ and hence

$$
A_{s}=a^{*}\left(e^{-i s \omega} f_{1}\right) a^{*}\left(e^{-i s \omega} f_{2}\right) \cdots a^{*}\left(e^{-i s \omega} f_{N}\right)
$$


Writing $W=a^{*}(G)+a(G)$ and using the canonical commutation relations, we thus have

$$
\begin{aligned}
{\left[W, A_{s}\right] } & =\left[a^{*}(G)+a(G), a^{*}\left(e^{-i s \omega} f_{1}\right) a^{*}\left(e^{-i s \omega} f_{2}\right) \cdots a^{*}\left(e^{-i s \omega} f_{N}\right)\right] \\
& =\left[a(G), a^{*}\left(e^{-i s \omega} f_{1}\right) a^{*}\left(e^{-i s \omega} f_{2}\right) \cdots a^{*}\left(e^{-i s \omega} f_{N}\right)\right] \\
& =\sum_{j=1}^{N}\left\{\left\langle G \mid e^{-i s \omega} f_{j}\right\rangle \otimes \prod_{\substack{i=1, i \neq j}}^{N} a^{*}\left(e^{-i s \omega} f_{i}\right)\right\}
\end{aligned}
$$

where $\left\langle G \mid e^{-i s \omega} f_{j}\right\rangle \in \mathcal{B}\left(\mathcal{H}_{e l}\right)$ is a bounded operator acting on the electron variables given by

$$
\left\langle G \mid e^{-i s \omega} f_{j}\right\rangle:=\int d^{3} k e^{-i s \omega(k)} f_{j}(k) G^{*}(k) .
$$

Note that $k \mapsto f_{j}(k) G^{*}(k)$ is $K>1$ times continuously differentiable, and, thanks to the support properties of $f_{j}$, there exists $r_{j}>0$ such that $f_{j} G^{*} \in C^{K}\left[B_{\Lambda} \backslash B_{r_{j}} ; \mathcal{B}\left(\mathcal{H}_{e l}\right)\right]$ has compact support away from zero. Thus, thanks to Hypothesis $1, K$ times $\left(\mathcal{B}\left(\mathcal{H}_{e l}\right)\right.$-valued) integration by parts yields the standard estimate for oscillatory integrals,

$$
\begin{aligned}
\| & (1+|x|)^{3 / 2}\left\langle G \mid e^{-i s \omega} f_{j}\right\rangle \| \\
& \leq|s|^{-K} \int d^{3} k\left\|(1+|x|)^{3 / 2}\left(\nabla_{k} \cdot(k /|k|)\right)^{K} f_{j}(k) G^{*}(k)\right\| \\
& \leq \mathcal{O}\left((1+|s|)^{-K}\right),
\end{aligned}
$$

provided $|s| \geq 1$. For $|s| \leq 1$, Estimate (III.20) is trivial and hence holds for all $s \in$ $\mathbb{R}$. Inserting (III.20) into (III.18) and undoing the free time evolution (which is possible because $H_{0}$ commutes with $H_{f}^{(\Lambda)}$ ), we obtain

$$
\begin{aligned}
\| & (1+|x|)^{3 / 2}\left[W, A_{s}\right]\left(H_{f}^{(\Lambda)}+\mathbf{1}\right)^{-1-(N / 2)} \| \\
\leq & \mathcal{O}\left((1+|s|)^{-K}\right) \sum_{j=1}^{N}\left\|\left(\prod_{\substack{i=1, i \neq j}}^{N} a^{*}\left(e^{-i s \omega} f_{i}\right)\right)\left(H_{f}^{(\Lambda)}+1\right)^{-1-(N / 2)}\right\| \\
& =\mathcal{O}\left((1+|s|)^{-K}\right) \sum_{j=1}^{N}\left\|\left(\prod_{\substack{i=1, i \neq j}}^{N} a^{*}\left(f_{i}\right)\right)\left(H_{f}^{(\Lambda)}+1\right)^{-1-(N / 2)}\right\| .
\end{aligned}
$$

To estimate a product of $N-1$ creation operators $\quad a^{*}\left(\tilde{f}_{1}\right) a^{*}\left(\tilde{f}_{2}\right) \cdots a^{*}\left(\tilde{f}_{N-1}\right)$ with $\tilde{f}_{1}, \tilde{f}_{2}, \ldots$,

$\tilde{f}_{N} \in C_{0}^{\infty}\left(B_{\Lambda} \backslash\{0\}\right)$, we use Estimate (III.4) $N-1$ times and derive

$$
\begin{aligned}
& \left\|\left(\prod_{n=1}^{N-1} a^{*}\left(\tilde{f}_{n}\right)\right)\left(H_{f}^{(\Lambda)}+\mathbf{1}\right)^{-(N-1) / 2)}\right\| \\
& =\left\|\prod_{n=1}^{N-1}\left(\left(H_{f}^{(\Lambda)}+\mathbf{1}\right)^{(n-1) / 2} a^{*}\left(\tilde{f}_{n}\right)\left(H_{f}^{(\Lambda)}+1\right)^{-n / 2}\right)\right\| \\
& \leq \prod_{n=1}^{N-1}\left\|\left(H_{f}^{(\Lambda)}+\mathbf{1}\right)^{(n-1) / 2} a^{*}\left(\tilde{f}_{n}\right)\left(H_{f}^{(\Lambda)}+1\right)^{-n / 2}\right\| \leq \mathcal{O}\left((1+\Lambda)^{\left(N^{2}\right)}\right) .
\end{aligned}
$$


Inserting this bound into (III.22) yields (III.16), since $\Lambda<\infty$ is bounded. $H_{f}^{(\Lambda)}$.

We finally turn to proving (II.11). Note that the following Lemma uses $H_{f}$ rather than

Lemma III.4. There exists a constant $C<\infty$ such that, for any $r \in \mathbb{R}$ and $s \in \mathbb{R}_{0}^{+}$,

$$
\left\|W e^{-i r H_{0}} P_{c}\left[W, A_{s}\right]\left(H_{f}+\mathbf{1}\right)^{-2-(N / 2)}\right\| \leq C\left((1+|r|)^{-3 / 2}\right) .
$$

Proof.

$e^{-i r(-\Delta)}(x, y)=(4 \pi i r)^{-3 / 2}$

The proof is based on the decay of the Schwartz kernel $\exp \left[i(x-y)^{2}(4 r)^{-1}\right]$ of the propagator $e^{-i r(-\Delta)}$ of the free particle. More precisely, defining $C_{1}:=(4 \pi)^{-1} \int(1+|x|)^{2 \gamma} d^{3} x<\infty$, we observe that

$$
\begin{aligned}
& \left|\left\langle(1+|x|)^{-\gamma} \varphi \mid e^{-i r(-\Delta)}(1+|x|)^{-\gamma} \psi\right\rangle\right| \\
& \quad=\frac{1}{(4 \pi r)^{3 / 2}}\left|\int d^{3} x d^{3} y \frac{\overline{\varphi(x)} e^{i(x-y)^{2} /(4 r)} \psi(y)}{(1+|x|)^{\gamma}(1+|y|)^{\gamma}}\right| \\
& \leq \frac{\|\varphi\|\|\psi\|}{(4 \pi r)^{3 / 2}}\left(\int \frac{d^{3} x}{(1+|x|)^{2 \gamma}}\right) \leq C_{1}\|\varphi\|\|\psi\| r^{-3 / 2},
\end{aligned}
$$

for any $\varphi, \psi \in L^{1} \cap L^{2}\left(\mathbb{R}^{3}\right)$, since $\gamma>3 / 2$. This estimate yields

$$
\begin{aligned}
& \left\|(1+|x|)^{-\gamma} e^{-i r H_{0}} P_{c}(1+|x|)^{-\gamma}\right\| \\
& \quad=\left\|\left(\begin{array}{cc}
(1+|x|)^{-\gamma} e^{-i r(-\Delta)}(1+|x|)^{-\gamma} & 0 \\
0 & 0
\end{array}\right) \otimes e^{-i r H_{f}}\right\| \leq C_{1} r^{-3 / 2} .
\end{aligned}
$$

Inserting (III.26) into (III.24) and using that $H_{f}$ and $H_{0}$ commute, we obtain

$$
\begin{gathered}
\left\|W e^{-i r H_{0}} P_{c}\left[W, A_{s}\right]\left(H_{f}+1\right)^{-2-(N / 2)}\right\| \\
\leq\left\|W(1+|x|)^{\gamma} \otimes\left(H_{f}+1\right)^{-1 / 2}\right\|\left\|(1+|x|)^{-\gamma} e^{-i r H_{0}} P_{c}(1+|x|)^{-\gamma}\right\| \\
\left\|(1+|x|)^{\gamma} \otimes\left(H_{f}+1\right)^{1 / 2}\left[W, A_{s}\right]\left(H_{f}+1\right)^{-2-(N / 2)}\right\| \\
\leq C_{1} r^{-3 / 2}\left\|W(1+|x|)^{\gamma} \otimes\left(H_{f}+1\right)^{-1 / 2}\right\| \\
\left\|(1+|x|)^{\gamma} \otimes\left(H_{f}+1\right)^{1 / 2}\left[W, A_{s}\right]\left(H_{f}+1\right)^{-2-(N / 2)}\right\| .
\end{gathered}
$$

Next, we use (III.18) and the pull-through formula to commute $\left(H_{f}+1\right)^{1 / 2}$ through $\left[W, A_{s}\right]$, observing that $\operatorname{supp} f_{j} \subseteq B_{\Lambda}(0)$. This yields

$$
\begin{aligned}
& \left\|(1+|x|)^{\gamma} \otimes\left(H_{f}+1\right)^{1 / 2}\left[W, A_{s}\right]\left(H_{f}+1\right)^{-2-(N / 2)}\right\| . \\
& \quad \leq\left\|(1+|x|)^{\gamma}\left[W, A_{s}\right]\left(H_{f}+1\right)^{-2-(N / 2)}\left(H_{f}+1+(N-1) \Lambda\right)^{1 / 2}\right\| \\
& \quad \leq(1+N \Lambda)^{1 / 2}\left\|(1+|x|)^{\gamma}\left[W, A_{s}\right]\left(H_{f}+1\right)^{-3 / 2-(N / 2)}\right\| \leq C_{2},
\end{aligned}
$$

for some constant $C_{2}<\infty$, uniformly in $s \in \mathbb{R}$. Here we additionally inserted (III.16) to derive the last inequality. Finally, there exists a constant $C_{3}<\infty$, such that

$$
\left\|W(1+|x|)^{\gamma} \otimes\left(H_{f}+1\right)^{-1 / 2}\right\| \leq C_{3},
$$

as follows from writing $W(1+|x|)^{\gamma}=a^{*}\left[G(1+|x|)^{\gamma}\right]+a\left[G(1+|x|)^{\gamma}\right]$ and applying Lemma III.1. 


\section{A Elimination of Matrix Elements by a Bogoliubov Trans- formation}

Consider $H_{e l}=-\Delta-V(x)$, a Schrödinger operator on $\mathbb{R}^{3}$ having a single, simple negative eigenvalue, $e_{0}<0$, associated to a normalized vector $\varphi_{0}$. This Hamiltonian represents an atom which we couple to the photonic field described in Subsect. I.1.2 with an interaction described in Subsect. I.1.3. More precisely, we assume the interaction $W$ to be of the form (I.11), with $G \in L^{2}\left[\mathbb{R}^{3} ; \mathcal{B}\left(\mathcal{H}_{e l}\right)\right]$ being a square-integrable function with values in the bounded operators on $\mathcal{H}_{e l}$, satisfying

$$
\int d^{3} k\left\{1+\omega(k)^{-2}\right\}\|G(k)\|^{2} \leq 1
$$

Note that this assumption is stronger than (I.16). We now show

Theorem A.1. Assume (A.1). For $g>0$ small enough, there exists a unitary transform $U_{g} \in \mathcal{B}\left(\mathcal{H}_{\text {el }} \otimes \mathcal{F}\right)$ real analytic in $g$ such that

$$
\widetilde{H}_{g}:=U_{g} H_{g} U_{g}^{*}=\widetilde{H}_{e l} \otimes \mathbf{1}_{f}+\mathbf{1}_{e l} \otimes H_{f}+g \widetilde{W}
$$

where

- $\widetilde{H}_{e l}=H_{e l}+g^{2} \Delta V$, and $\Delta V$ is a bounded self-adjoint operator;

- if $W$ acts as a multiplication operator in the electron variable, $\Delta V$ is a potential, i.e., a multiplication operator;

- $\widetilde{H}_{e l}$ has a single, simple negative eigenvalue, $e_{g}<0$, associated to the one-dimensional eigenprojector $\Pi_{g}$;

- we have

$$
\left(\Pi_{g} \otimes \mathbf{1}_{f}\right) \widetilde{W}\left(\Pi_{g} \otimes \mathbf{1}_{f}\right)=0
$$

All the quantities introduced above are real-analytic in $g$.

Theorem A.1 proves that, starting from a Schrödinger operator on $\mathbb{R}^{3}$ with a single, simple negative bound state, using a unitary transform, one can always pass to an interaction fulfilling Assumption (I.12).

Proof. Pick $h \in L^{2}\left(\mathbb{R}^{3}\right)$, and consider the Bogoliubov transform

$$
U(h)=\mathbf{1} \otimes e^{i g\left[a^{*}(h)+a(h)\right]} .
$$

A standard computation gives

$$
U(h) H_{g} U(h)^{*}=\widetilde{H}_{e l} \otimes \mathbf{1}_{f}+\mathbf{1}_{e l} \otimes H_{f}+g \widetilde{W},
$$


where

$$
\begin{aligned}
\widetilde{H}_{e l} & =H_{e l}+g^{2} \Delta V(h), \\
\Delta V(h) & :=\int d^{3} k\left\{h(k) G(k)+\overline{h(k)} G^{*}(k)\right\}, \\
\widetilde{W} & =W+\mathbf{1} \otimes\left\{a^{*}(\omega h)+a(\omega h)\right\} .
\end{aligned}
$$

We remark that $\Delta V: L^{2}\left(\mathbb{R}^{3}\right) \rightarrow \mathcal{B}\left(\mathcal{H}_{e l}\right)$ is a bounded linear operator with norm bounded by one, thanks to (A.1). Hence, $\widetilde{H}_{e l}$ has a single, simple eigenvalue $\tilde{e}(h)=e_{0}+\mathcal{O}\left(g^{2}\right)$ in a vicinity of $e_{0}$, and we denote the corresponding normalized eigenvector by $\varphi_{g}(h)$.

To prove Theorem A.1, we only need to show that, for $g>0$ sufficiently small, we can construct $h_{g} \in L^{2}\left(\mathbb{R}^{3}\right)$ such that

$$
-\left\langle\varphi_{g}\left(h_{g}\right), \widehat{G}(k) \varphi_{g}\left(h_{g}\right)\right\rangle=h_{g}(k), \quad \text { where } \quad \widehat{G}(k):=\omega(k)^{-1} G(k) .
$$

By Assumption (A.1), $\widehat{G}$ is a square-integrable function with values in the bounded operators on $\mathcal{H}_{e l}$. Using standard perturbation theory, we construct $\varphi_{g}(h)$ simply by normalizing the vector

$$
\hat{\varphi}_{g}(h):=\frac{1}{2 i \pi} \int_{\gamma_{0}}\left(z-H_{e l}-g^{2} \Delta V(h)\right)^{-1} \varphi_{0} d z
$$

where $\gamma_{0}$ is a small circle of center $e_{0}$ and radius $\left|e_{0} / 2\right|$. The function $\varphi_{g}(h)$ is real analytic in $g$ and continuous in $h$, for $h$ in the unit ball in $L^{2}\left(\mathbb{R}^{3}\right)$; real analytic perturbation theory and the bound $\|\Delta V(h)\| \leq\|h\|$ immediately give

$$
\left\|\varphi_{g}(h)-\varphi_{g}\left(h^{\prime}\right)\right\| \leq C g^{2}\left\|h-h^{\prime}\right\|,
$$

for some constant $C<\infty$ and all $h, h^{\prime} \in L^{2}\left(\mathbb{R}^{3}\right)$ with $\|h\|,\left\|h^{\prime}\right\| \leq 1$. Set

$$
h_{0}=-\left\langle\varphi_{0} \mid \widehat{G}(k) \varphi_{0}\right\rangle \text {. }
$$

For $g$ fixed and small and $h \in L^{2}\left(\mathbb{R}^{3}\right)$, with $\|h\| \leq 1$, we define

$$
T_{g}(h)=-\left\langle\varphi_{g}\left(h_{0}+h\right) \mid \widehat{G}(\cdot) \varphi_{g}\left(h_{0}+h\right)\right\rangle-h_{0} .
$$

Note that $T_{0}$ has a trivial fixed point, $h=0$. For $g>0$ sufficiently small, $T_{g}$ maps the unit ball of $L^{2}\left(\mathbb{R}^{3}\right)$ into itself. Moreover, one computes

$$
\begin{aligned}
\left\|T_{g}(h)-T_{g}\left(h^{\prime}\right)\right\| & \leq 4\|\widehat{G}(k)\|\left\|\varphi_{g}\left(h_{0}+h\right)-\varphi_{g}\left(h_{0}+h^{\prime}\right)\right\| \\
& \leq C g^{2}\left\|h-h^{\prime}\right\|,
\end{aligned}
$$

for some $C<\infty$. Hence, $T_{g}$ is contracting for $g$ small enough. Therefore, the fixed point equation $h=T_{g}(h)$ has a unique solution in the unit ball of $L^{2}\left(\mathbb{R}^{3}\right)$. This fixed point is the desired function solving (A.9). We may construct this solution as the norm limit of the sequence $T_{g}^{n}(0)$. Each of these terms being real analytic in $g$ and the convergence being uniform in $g$ sufficiently small (the rate of convergence is given by $g^{2}$ ), the limit is real analytic in $g$. 


\section{B Transported Charge for Bound and Negative Energy States}

Theorem B.1. Assume Hypothesis 1. Then

$$
\begin{aligned}
\lim _{R \rightarrow \infty} \sup _{t \geq 0}\left\|F_{R} e^{-i t H_{g}} \mathbf{1}_{p p}\left(H_{g}\right) \Psi\right\| & =0 \\
\lim _{R \rightarrow \infty} \sup _{t \geq 0} \| F_{R} e^{-i t H_{g}} & \mathbf{1}_{]-\infty, 0]}\left(H_{g}\right) \Psi \|=0
\end{aligned}
$$

for any $\Psi \in \mathcal{H}$, where $\mathbf{1}_{p p}\left(H_{g}\right)$ and $\mathbf{1}_{]-\infty, 0]}\left(H_{g}\right)$ are the spectral projections of $H_{g}$ onto its point spectrum and onto ] $-\infty, 0]$, respectively.

Proof. To prove equality (B.1), we first assume that $\Psi$ is an eigenvector of $H_{g}$ with corresponding eigenvalue $E \in \mathbb{R}$. Since $F_{R} \rightarrow 0$ strongly, as $R \rightarrow \infty$, we then have

$$
\lim _{R \rightarrow \infty} \sup _{t \geq 0}\left\|F_{R} e^{-i t H_{g}} \Psi\right\|=\lim _{R \rightarrow \infty}\left\|F_{R} \Psi\right\|=0 .
$$

This, of course, generalizes to any finite linear combination of eigenvectors of $H_{g}$. Since the norm closure of this set of vectors is the pure point subspace of $H_{g}$, Eq. (B.3) also generalizes to vectors in $\operatorname{Ran} \mathbf{1}_{p p}\left(H_{g}\right)$, which yields (B.1). As a consequence of (B.1) we may assume $\Psi=1_{c}\left(H_{g}\right) \Psi$ in the proof of (B.2). These vectors out of the continuous subspace have the property that

$$
\lim _{\delta \searrow 0} \mathbf{1}_{(E-\delta, E+\delta)}\left(H_{g}\right) \Psi=0
$$

for any $E \in \mathbb{R}$. As $H_{g}$ is bounded from below and in view of (B.4), it hence (choosing $E:=0$ ) suffices to prove that

$$
\lim _{R \rightarrow \infty}\left\|F_{R} \chi\left[H_{g}\right]\right\|=0,
$$

for any smooth function $\chi \in C_{0}^{\infty}[(-\infty, 0)]$, compactly supported on the negative half-axis and away from zero. The basic idea of our proof of (B.5) is essentially the same as [2, Thm. II.1] or Lemma III.2 and uses a Combes-Thomas or Agmon Estimate. For $H=H_{g}$ or $H=H_{0}$, we use a representation

$$
\chi[H]=\int_{\Delta} \frac{d \mu(z)}{H-z}
$$

based on an almost analytic extension $\tilde{\chi} \in C_{0}^{\infty}(\mathcal{M} ; \mathbb{C})$ of $\chi$, whose compact support $\mathcal{M} \subseteq \mathbb{C}$ can be chosen to include only $z \in \mathbb{C}$ with $\operatorname{Re} z \leq-\delta$, for some $\delta>0$. Moreover, we can choose $\tilde{\chi}$ as to obey $\bar{\partial} \tilde{\chi}(z)=\mathcal{O}\left((\operatorname{Im} z)^{2}\right)$. The measure in (B.6) is then defined as $d \mu(z):=(2 \pi i)^{-1} \bar{\partial} \tilde{\chi}(z) d z \wedge d \bar{z}$. By means of (B.6), the second resolvent equation, and the fact that $F_{R} \chi\left[H_{0}\right]=F_{R} P_{c} \chi\left[H_{0}\right]=0$, since $H_{0} \geq 0$ on Ran $P_{c}$, we thus have

$$
\begin{aligned}
F_{R} \chi\left[H_{g}\right] & =F_{R}\left(\chi\left[H_{g}\right]-\chi\left[H_{0}\right]\right) \\
& =-g \int_{\mathcal{M}} d \mu(z) F_{R} \frac{P_{c}}{-\Delta+H_{f}-z} W \frac{1}{H_{g}-z} .
\end{aligned}
$$


Next, we pick $1<\lambda<\infty$ and introduce $f_{\lambda}: \mathbb{R}^{3} \rightarrow \mathbb{R}^{+}$by $f_{\lambda}(x):=\sqrt{1+(x / \lambda)^{2}}$, and we denote $f_{\lambda}(x)^{-1}:=1 / f_{\lambda}(x)$. We note the following properties of $f_{\lambda}$,

$$
\frac{|x|}{\lambda} \leq f_{\lambda}(x) \leq 1+|x|^{3 / 2} \quad \text { and } \quad f_{\lambda}(x)^{-1}\left|\nabla f_{\lambda}(x)\right| \leq \lambda^{-1}
$$

which imply

$$
\begin{aligned}
\left\|F_{R} f_{\lambda}(x)^{-1}\right\| & \leq C \lambda R^{-1} \\
\operatorname{Re}\left\{f_{\lambda}(x)(-\Delta) f_{\lambda}(x)^{-1}\right\} & = \\
-\Delta+2 \operatorname{Re}\left\{f_{\lambda}(x)^{-1} \nabla f_{\lambda}(x) \cdot i \nabla\right\} & \geq-\lambda^{-2} \\
\left\|f_{\lambda}(x) W\left(H_{g}+i\right)^{-1}\right\| & \leq C
\end{aligned}
$$

for some constant $C<\infty$. Here, (B.9) is trivial, (B.10) is meant in the quadratic form sense on smooth functions of compact support, and (B.11) uses Hypothesis 1 and Lemma III.1. Choosing $\lambda \geq 2 / \sqrt{\delta}$ and inserting the bounds (B.9)-(B.11) into (B.7), we arrive at (B.5).

\section{References}

[1] W. Amrein, A. Boutet de Monvel, and V. Georgescu. $C_{0}$-Groups, Commutator Methods, and Spectral Theory of N-Body Hamiltonians. Birkhäuser, 1996.

[2] V. Bach, J. Fröhlich, and I. M. Sigal. Quantum electrodynamics of confined nonrelativistic particles. Adv. in Math. , 137:299-395, 1998.

[3] V. Bach, J. Fröhlich, and I. M. Sigal. Renormalization group analysis of spectral problems in quantum field theory. Adv. in Math. , 137:205-298, 1998.

[4] V. Bach, J. Fröhlich, and I. M. Sigal. Spectral analysis for systems of atoms and molecules coupled to the quantized radiation field. Commun. Math. Phys., 207(2):249-290, 1999.

[5] V. Bach, J. Fröhlich, and I. M. Sigal. Return to equilibrium. J. Math. Phys., 41(6):3985-4060, June 2000.

[6] J. Derezinski and C. Gérard. Scattering theory of classical and quantum N-particle systems. Text and Monographs in Physics. Springer, 1997.

[7] J. Derezinski and C. Gérard. Asymptotic completeness in quantum field theory. Massive Pauli-Fierz Hamiltonians. Rev. Math. Phys., 11:383-450, 1999

[8] D. Eastham. Atomic Physics of Lasers. Taylor and Francis, London, 1986.

[9] A. Einstein. Über einen die Erzeugung und Verwandlung des Lichts betreffenden heuristischen Gesichtspunkt. Annalen der Physik, 17:132-148, 1905.

[10] J. Fröhlich, M. Griesemer, and B. Schlein. Asymptotic completeness for Rayleigh scattering. Preprint, 2001. 
[11] J. Fröhlich, M. Griesemer, and B. Schlein. Asymptotic completeness for Compton scattering. Preprint, 2001.

[12] Ch. Gérard. Asymptotic completeness for the spin-boson model with a particle number cutoff. Rev. Math. Phys., 8:549-589, 1996.

[13] M. Griesemer, E. Lieb, and M. Loss. Ground states in nonrelativistic quantum electrodynamics. Invent. math., June 2001. DOI 10.1007/s002220100159.

[14] H. Haken. Light, volume 1. North-Holland, 1981.

[15] D. Haliday and R. Resnick. Physics (Part I and II). Wiley, 1978.

[16] W. Hallwachs. Ueber den Einfluss des Lichtes auf electrostatisch geladene Körper. Annalen der Physik, 33:301, 1888.

[17] H. Hertz. Ueber einen Einfluss des ultravioletten Lichtes auf die electrische Entladung. Annalen der Physik, 31:983, 1887.

[18] P. Lenard. Über die lichtelektrische Wirkung. Annalen der Physik, 8:149-198, 1902.

[19] A. Melin and J. Sjöstrand. Fourier Integral Operators with Complex-valued Phase Functions, volume 459 of L. N. M. . Springer, Heidelberg, 1976.

[20] R. Millikan. A direct photoelectric determination of Planck's h. Phys. Rev., 7:355$388,1916$.

[21] R. Millikan. Einstein's photoelectric equation and contact electromotive force. Phys. Rev., 7:18-32, 1916.

[22] M. Reed and B. Simon. Methods of modern mathematical physics. III. Academic Press, New York, 1979. Scattering theory.

[23] B. Schlein. Nichtrelativistische Elektronensysteme in einem quantisierten elektromagnetischen Feld. diploma thesis, ETH-Zürich, August 1999. 